\title{
Unlocking the Full Potential of Extragalactic Ly $\alpha$ through Its Polarization Properties
}

\author{
Marius B. Eide ${ }^{1}(1)$, Max Gronke $^{2,3}$ (1) , Mark Dijkstra $^{2}$, and Matthew Hayes ${ }^{4}(1)$ \\ ${ }^{1}$ Max-Planck-Institut für Astrophysik, Karl-Schwarzschild-Straße 1, D-85741 Garching, Germany; eide@MPA-Garching.MPG.DE \\ ${ }^{2}$ Institute of Theoretical Astrophysics, University of Oslo, Postboks 1029, NO-0315 Oslo, Norway \\ ${ }^{3}$ Department of Physics, University of California, Santa Barbara, CA 93106, USA \\ ${ }^{4}$ Department of Astronomy, Oskar Klein Centre, Stockholm University, AlbaNova University Centre, SE-106 91 Stockholm, Sweden \\ Received 2017 September 13; revised 2018 February 27; accepted 2018 March 7; published 2018 April 4
}

\begin{abstract}
Ly $\alpha$ is a powerful astrophysical probe. Not only is it ubiquitous at high redshifts, it is also a resonant line, making Ly $\alpha$ photons scatter. This scattering process depends on the physical conditions of the gas through which Ly $\alpha$ propagates, and these conditions are imprinted on observables such as the Ly $\alpha$ spectrum and its surface brightness profile. In this work, we focus on a less-used observable capable of probing any scattering process: polarization. We implement the density matrix formalism of polarization into the Monte Carlo radiative transfer code tlac. This allows us to treat it as a quantum mechanical process where single photons develop and lose polarization from scatterings in arbitrary gas geometries. We explore static and expanding ellipsoids, biconical outflows, and clumpy multiphase media. We find that photons become increasingly polarized as they scatter and diffuse into the wings of the line profiles, making scattered Ly $\alpha$ polarized in general. The degree and orientation of Ly $\alpha$ polarization depends on the kinematics and distribution of the scattering H I gas. We find that it generally probes spatial or velocity space asymmetries and aligns itself tangentially to the emission source. We show that the mentioned observables, when studied separately, can leave similar signatures for different source models. We conclude by revealing how a joint analysis of the Ly $\alpha$ spectra, surface brightness profiles, and polarization can break these degeneracies and help us extract unique physical information on galaxies and their environments from their strongest, most prominent emission line.
\end{abstract}

Key words: galaxies: halos - galaxies: high-redshift - galaxies: ISM - line: formation - polarization - radiative transfer

\section{Introduction}

Ly $\alpha$ is the torch that lights up the distant universe. Partridge \& Peebles (1967) recognized Ly $\alpha$ to be the strongest tracer of recombining ionized hydrogen (H II) in young, (star-) forming galaxies. However, the search for redshifted Ly $\alpha$ emission was fruitless until the late 1980s, when Ly $\alpha$ finally was found in known radio galaxies (see, e.g., Djorgovski et al. 1985, Hu \& Cowie 1987, or the overview by Spinrad 1989). Today, Ly $\alpha$ heralds the presence of the most distant sources known to humankind (e.g., through absorption, Oesch et al. 2016; or emission, Zitrin et al. 2015), and detecting Ly $\alpha$ has become one of the primary science goals of future instruments and telescopes that are developed to understand the high- $z$ universe (see, e.g., reviews by Dijkstra 2014 and Hayes 2015).

The question remains as to what one can learn from observations of Ly $\alpha$ emission (and/or absorption). Thus far, observational efforts, as well as theoretical advances geared toward Ly $\alpha$ radiation, have focused primarily on the modulation of intensity. However, Ly $\alpha$ radiation (or radiation of any wavelength) possesses two more degrees of freedom, ${ }^{5}$ which quantify its polarization properties. These are often represented through the Stokes $Q$ and $U$ parameters and give the direction and degree of polarization. In this work, we explore what additional knowledge can be obtained from these observables.

The potential power of Ly $\alpha$ lies in its resonance nature. In contrast to $\mathrm{H} \alpha$, which escapes unobstructed from its production site following recombination, a Ly $\alpha$ photon can undergo a

\footnotetext{
5 A third degree of freedom also exists for circularly polarized light: the timedependence of the polarization angle, expressed through the Stokes $V$ parameter.
}

tremendous number of scatterings after creation, where the precise number depends on $\mathrm{HI}$ column density, geometry, and kinematics (Adams 1972; Dijkstra 2014). Each scattering event results in a slight change in position and frequency. This dual diffusion process (Osterbrock 1962) imprints signatures on the emergent observables and potentially reveals properties of the scattering medium along the paths that offered least resistance to the photons (see, e.g., Dijkstra et al. 2016; Gronke \& Dijkstra 2016).

These signatures can also act as keys to uncovering the emission mechanism. Centrally emitted Ly $\alpha$ photonse.g., when they were created as nebular emission powered by Pop II stars (Chapman et al. 2004), Pop III stars (Schaerer 2002, 2003), or a nuclear black hole ${ }^{6}$ (Geach et al. 2009)-must scatter significantly in most cases prior to escape. Spatially extended Ly $\alpha$ emission can be produced by inflowing, cooling gas (Haiman et al. 2000), gas that has been shock heated by supernova explosions (Mori et al. 2004) or galactic superwinds (Taniguchi \& Shioya 2000), or as fluorescent radiation from an external ionizing field (Hogan \& Weymann 1987; Cantalupo et al. 2005). These photons do not need to escape from the dense insterstellar medium (ISM) and therefore typically scatter less. With $\operatorname{Ly} \alpha$ ubiquitously present in galaxies, surveys provide a wealth of observations open for interpretation (e.g., Steidel et al. 2011; Wisotzki et al. 2016; Herenz et al. 2017; Shibuya et al. 2018). Theoretical work exploring the modulation of $\operatorname{Ly} \alpha$ observables by radiative transfer effects aims to convert these observables

\footnotetext{
6 Such spectrally hard sources would leave notably large Ly $\alpha$ equivalent widths.
} 
into constraints on the physical conditions of the gas in and around galaxies.

Currently, two quantities provide the main observables. One is the spectrum, which encodes information on the frequency diffusion process of the photons, leading to broadening and shifting of the spectral line shape (Neufeld 1990; Dijkstra et al. 2006) by an amount that depends on kinematics, geometry, and dust content of the scattering medium (e.g., Ahn \& Lee 1998; Hansen \& Peng Oh 2006; Verhamme et al. 2006; Dijkstra \& Loeb 2008; Gronke et al. 2015). These models have been successful at reproducing observations (Verhamme et al. 2008; Hashimoto et al. 2015; Karman et al. 2016; Yang et al. 2017), though it still unclear how physically realistic they are (see, e.g., Gronke \& Dijkstra 2016; Gronke et al. 2016, 2017).

One problem is that widely different models can provide similar spectra. For example, the large majority of emission sources have a Ly $\alpha$ peak that is redshifted with respect to other lines in the system (Kunth et al. 1998; Trainor et al. 2015), something that can be easily explained by $\operatorname{Ly} \alpha$ scattering through a galactic outflow (Dijkstra et al. 2006; Verhamme et al. 2006). However, it is known that the intergalactic medium (IGM) can also process away $\operatorname{Ly} \alpha$, mainly in the blue part of the intensity spectrum, which can leave an intrinsically symmetric emission line with a net redshift (Dijkstra et al. 2007; Laursen et al. 2011). Dijkstra \& Loeb (2008) showed that these different models give rise to different levels of polarization. This illustrates that polarization, when combined with spectroscopy, may tell models apart that otherwise are indistinguishable.

The other quantity, the surface brightness profile, can reveal the spatial diffusion process that $\operatorname{Ly} \alpha$ photons undergo before escaping, possibly far from the site of emission. The resulting Ly $\alpha$ nebulae have been detected around many Ly $\alpha$-emitting galaxies (Hayes et al. 2013; Wisotzki et al. 2016), with larger counterparts around many quasars (Cantalupo et al. 2014; Hennawi et al. 2015; Lake et al. 2015; Cai et al. 2017), but not all (Herenz et al. 2015).

With integral field unit spectrometers (IFUs) such as MUSE (Bacon et al. 2015) or deep-imaging surveys as SILVERRUSH (Ouchi et al. 2017), the number of detailed Ly $\alpha$ observationsthat is, spectra and sometimes surface brightness informationexceeds thousands. There are far fewer observations of polarized Ly $\alpha$ (Hayes et al. 2011; Prescott et al. 2011; Humphrey et al. 2013; Beck et al. 2016; You et al. 2017). This is partially due to the observational difficulty associated with polarization measurements of distant sources. Polarizationequipped instruments presently have small fields of view, and multiplexed observations of the Stokes parameters are generally hard. However, another reason is a lack of theoretical foundation, which makes $\operatorname{Ly} \alpha$ polarization results difficult to interpret. This is something we wish to improve upon with this work.

Presently, there are two ways of implementing polarized Ly $\alpha$ transfer in numerical codes. The first approach treats polarization solely in the macroscopical sense and assumes that all photons are 100\% linearly polarized by "carrying" a polarization vector in addition to a direction vector and frequency (Angel 1969; Rybicki \& Loeb 1999; Dijkstra \& Loeb 2008; Trebitsch et al. 2016). The second approach is that of Lee et al. (1994; also used in Lee \& Blandford 1997; Lee \& Lee 1997; Lee \& Ahn 1998; Lee 1999; Ahn \& Lee 2015;
Chang et al. 2017), who employed a quantum mechanically precise treatment of scattering and polarization using density matrices, allowing unpolarized photons to develop polarization through scatterings (and allowing polarized photons to become depolarized). We employ this latter method, as it is quantum mechanically more accurate, and implement it in the Monte Carlo radiative transfer code tlac (Gronke \& Dijkstra 2014).

The goal of this paper is to explore what additional information is encoded within the polarization properties of Ly $\alpha$ on the physical properties of the scattering medium. More concretely, our goal is to go beyond the "standard" predictions for intensity $I$ and focus on two linear polarization parameters, $Q$ and $U$, and see whether this extra information can break degeneracies between different models in order to gain a deeper physical understanding of sources of Ly $\alpha$.

This work is structured as follows. We describe the detailed numerical implementation of the density matrix formalism of Ly $\alpha$ polarization in Section 2. This section is technical and can be skipped by readers who are mostly interested in the results, which we present in Section 3. We discuss our results in more detail and in a broader context in Section 4, before concluding in Section 5.

\section{Ly $\alpha$ Monte Carlo Polarization}

Radiative transfer is the art of describing the complex and arduous journey light takes after being emitted. The equation of radiative transfer ${ }^{7}$ illustrates this: a change in intensity at one frequency $\nu$ along a differential path length is affected by three factors: (1) attenuation, (2) emission, and (3) redistribution in both space and frequency. The third factor is of paramount importance for Ly $\alpha$. It expresses any contributions to the intensity that did not originate at the same frequency or from the same direction. It is thus an integral over all frequencies and all solid angles embedded in a differential equation.

Monte Carlo methods are the preferred way of treating radiative processes where the photons do not alter the state of the medium they travel through but are still sensitive to the redistributions caused by scattering through it (see, e.g., Avery \& House 1968; Lee \& Lee 1997; Loeb \& Rybicki 1999; Ahn et al. 2000; Zheng \& Miralda-Escudé 2002; Dijkstra \& Loeb 2008; Pierleoni et al. 2009; Laursen 2010) becausealbeit slow-they guarantee convergence even in complex density or velocity fields.

Here we describe the basics of polarization in Sections 2.1-2.3 and how we implement the density matrix formalism into the radiative transfer code tlac (Gronke \& Dijkstra 2014) in Section 2.4.

\subsection{Emission}

Ly $\alpha$ photons are emitted at or near the $\mathrm{Ly} \alpha$ resonance frequency of $\nu_{0}=2.47 \times 10^{15} \mathrm{~Hz}$ for hydrogen. We parameterize their offset from the line center through $x \equiv\left(\nu-\nu_{0}\right) / \Delta \nu_{\mathrm{D}}$, where the Doppler width is defined as $\Delta \nu_{\mathrm{D}}=v_{\mathrm{th}} \nu_{0} / c$ with the thermal velocity $v_{\mathrm{th}}=\sqrt{2 k_{\mathrm{B}} T_{g} / m_{p}}$, which depends on the gas temperature $T_{g}$, as well as Boltzmann's constant $k_{\mathrm{B}}$ and the proton mass $m_{p}$. The speed of light is $c$. We also parameterize the offset from the line center in terms of the thermal velocity. The relationship is $v=-\lambda_{0} \Delta \nu_{\mathrm{D}} x$. We also represent the spread in emission

\footnotetext{
For instance, given by Equation (1) in the review by Dijkstra (2014) in its differential form.
} 


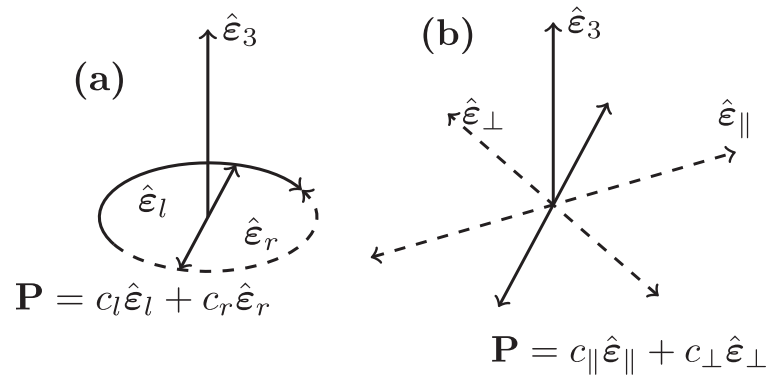

Figure 1. Conceptual sketches of two possible choices of bases in the plane perpendicular to the photon propagation direction, which is chosen to be $\hat{\varepsilon}_{3}$ : (a) complex, helical coordinates representative of the intrinsic spin of the photon, and (b) Cartesian coordinates representative of linear polarization appearing from a photon being in both spin states and having a fixed, nonvarying phase between the helical spins. The oscillations of the complex polarization vector $\boldsymbol{P}$ in the plane are also drawn.

around the line center as the standard deviation of a Gaussian with $\sigma_{i}$ in units of $\mathrm{km} \mathrm{s}^{-1}$.

The photons are massless and have four degrees of freedom through their two spins. Measuring the spin is synonymous with measuring the polarization. Intrinsically, the photons possess helical spins, whereas observationally, it is advantageous to consider the linear representation instead and include a possible phase relation.

Following the approach of Lee et al. (1994), we construct a complex state vector $\boldsymbol{P}$ with four degrees of freedom, represented through the complex coefficients $c_{1}$ and $c_{2}$, given in an orthogonal basis $\left\{\hat{\varepsilon}_{1}, \hat{\varepsilon}_{2}, \hat{\varepsilon}_{3}\right\}$ (where $\hat{\varepsilon}_{3}$ denotes the propagation direction) as

$$
\boldsymbol{P}=c_{1} \hat{\varepsilon}_{1}+c_{2} \hat{\varepsilon}_{2} .
$$

For a given $\boldsymbol{P}$, the values of the coefficients $c_{1}$ and $c_{2}$ depend on the choice of basis, which is determined by what we desire to observe: circular or linear polarization. For example, a helical basis is best suited to describe circular polarization, and we may use $c_{1} \hat{\varepsilon}_{1}=c_{l} \hat{\varepsilon}_{l}$ and $c_{2} \hat{\varepsilon}_{2}=c_{r} \hat{\varepsilon}_{r}$ to represent the left- and righthanded components of the spin, with probabilities $\left|c_{l}^{2}\right|,\left|c_{r}^{2}\right|$ of finding the photon to be either left- or right-handed polarized, respectively. On the other hand, linear polarization arises from the superposition of the helical spins, describing it in terms of a parallel and perpendicular component; these can be written as $c_{\|} \hat{\varepsilon}_{\|}=\left(c_{l} \hat{\varepsilon}_{l}+c_{r} \hat{\varepsilon}_{r}\right) / \sqrt{2}$ and $c_{\perp} \hat{\varepsilon}_{\perp}=i\left(c_{l} \hat{\varepsilon}_{r}-c_{r} \hat{\varepsilon}_{r}\right) / \sqrt{2}$, respectively (see Figure 1 for a conceptual sketch of these representations of the polarization).

We may ask: if the squares of the coefficients are equally large, is a polarization signal observable? The answer lies in the phase delay between the components: if both coefficients have equal magnitude and no fixed phase delay exists, there is no polarization. If there is a phase delay, however, we will obtain a polarization signal. These additional constraints may be obtained from the cross-terms $c_{1} c_{2}^{*}$ and $c_{1}^{*} c_{2}$. This discussion illustrates that the density matrix of the photon, $\rho_{\text {phot }}$, contains all information on its quantum state,

$$
\rho_{\mathrm{phot}}=\boldsymbol{P P}^{\dagger}=\left(\begin{array}{ll}
c_{1} c_{1}^{*} & c_{1} c_{2}^{*} \\
c_{2} c_{1}^{*} & c_{2} c_{2}^{*}
\end{array}\right),
$$

where the off-diagonal elements give the time-dependent phase between the two states, and the diagonal elements give the probabilities of measuring the photon in either of the two states.

\subsection{Scattering}

After emission, the photons may scatter with neutral hydrogen gas particles. ${ }^{8}$ This interaction excites the particle from its ground state to an intermediate state, which it immediately de-excites into its final state. Should the initial and final states be the same, the photon will neither gain nor lose any energy. ${ }^{9}$ However, for our usage cases, these effects can be ignored (Adams 1971), and the scattering is elastic. We will not treat inelastic Raman scattering but rather refer the interested reader to Lee \& Lee (1997) for an in-depth study of the polarization properties of Raman-scattered light.

When a Ly $\alpha$ photon elastically scatters, it experiences three types of redistribution: (i) change in propagation direction, (ii) change of frequency, and (iii) change of polarization. We discuss each below.

1. Change of propagation direction. The change of direction is quantified by the phase function, which we denote with $p\left(\theta^{\prime}, \phi^{\prime} \mid \rho_{\text {phot }}, \theta, \phi\right)$. Primed quantities denote scattered values. As we describe below, this phase function depends on the frequency and polarization of a Ly $\alpha$ photon. It gives the probability of a photon being in the state it would obtain following a scattering and can be directly related to the density matrix,

$$
p\left(\theta^{\prime}, \phi^{\prime} \mid \rho_{\text {phot }}, \theta, \phi\right)=\frac{\left|c_{1}^{2^{\prime}}\right|+\left|c_{2}^{2^{\prime}}\right|}{\int\left|c_{1}^{2^{\prime}}\right|+\left|c_{2}^{2^{\prime}}\right| d \Omega} .
$$

Each scattered density matrix component is obtained by a linear combination of the three incoming components, with trigonometric functions weighing each contribution. Expressions for the density matrix are complex (see, e.g., Lee et al. 1994 or Lee 1994 for prescriptions for obtaining them or Ahn \& Lee (2015) for the relevant expressions for $\operatorname{Ly} \alpha$ ), and we refer interested readers to these papers. Full expressions for $c_{1}^{2^{\prime}}$ and $c_{2}^{2^{\prime}}$ as a function of $\left(\theta^{\prime}, \phi^{\prime}, \rho_{\text {phot }}, \theta, \phi\right)$ are given in Appendix A.

2. Change of frequency. The "type" of elastic scattering depends on the offset from the resonance frequency $\nu_{0}$, as seen from the scattering atom. We express the velocity $v_{\text {atom }}$ of the scattering atom as a dimensionless velocity $\boldsymbol{u}=\boldsymbol{v}_{\text {atom }} / v_{\text {th }}$. The frequency shift of the Ly $\alpha$ photon in the rest frame of the atom, $x_{e}$, is then

$$
x_{e}=x_{i}-\boldsymbol{u} \cdot \hat{\boldsymbol{k}}
$$

where $x_{i}$ is the initial frequency shift (Laursen 2010). We can differentiate between resonance scattering $\left(x_{e} \sim 0\right)$ and wing scattering $\left(\left|x_{e}\right| \gg 0\right)$. This distinction is important: Stenflo (1980) showed that, for resonant scattering, the polarization properties of scattered Ly $\alpha$ relate to the spin properties of the atomic configuration of the $\mathrm{H}$ atom. On the other hand, for wing scattering, the electron behaves as if it is free.

The transition from core to wing occurs at a temperature-dependent frequency offset $x_{c w} \sim 3$ (see, e.g., Laursen 2010 for an expression for $x_{c w}$ ). We also use

\footnotetext{
8 In this work, we focus exclusively on scattering by $\mathrm{H}$ I atoms. Scattering by dust and electrons can be included in future studies. However, although dust clearly plays an important role in the Ly $\alpha$ radiative transfer process, its effect is mostly to destroy Ly $\alpha$ photons.

9 This is not entirely true. The photons deposit and gain energy through atomic recoil (Madau et al. 1997) and hyperfine excitation of the ground state (Wouthuysen 1952; Field 1958).
} 
an acceleration scheme for $\operatorname{Ly} \alpha$ Monte Carlo radiative transfer as in Dijkstra et al. (2006) but have explicitly verified that our results are not affected by this.

3. Change of polarization. The change of polarization properties is quantified by the change of the density matrix per scattering event. The newly obtained total ${ }^{10}$ degree of polarization of a photon $P_{\text {phot }}$ following a scattering is the fraction of the linearly $(Q$ and $U$ ) and circularly $(V)$ polarized intensity to the total intensity $(I)$,

$$
\begin{gathered}
P_{\text {phot }}\left(\theta^{\prime}, \phi^{\prime} \mid \rho_{\text {phot }}, \theta, \phi\right)=\frac{\sqrt{Q^{2}+U^{2}+V^{2}}}{I} \\
=\frac{\sqrt{\left(\left|c_{1}^{2^{\prime}}\right|-\left|c_{2}^{2^{\prime}}\right|\right)^{2}+2^{2}\left(c_{1} c_{2}^{*}\right)^{\prime}\left(c_{1}^{*} c_{2}\right)^{\prime}}}{\left|c_{1}^{2^{\prime}}\right|+\left|c_{2}^{2^{\prime}}\right|},
\end{gathered}
$$

following Ahn \& Lee (2015).

We now turn to discussing resonant and wing scattering in more detail, as the distinction between the two plays an important role in the above processes.

\subsubsection{Resonance Scattering}

For $\left|x_{e}\right|<x_{\mathrm{cw}}$, we will consider scatterings dominated by the transition from the ground energy state of hydrogen, denoted ${ }^{11}$ $1 S_{1 / 2}$, to the excited $n=2$ state, comprising the two available orbital configurations $2 S_{1 / 2}$ or $2 P_{J}$, where the $2 P_{J}$ level is degenerate into $J=1 / 2$ and $J=3 / 2$ and back to the final $1 S_{1 / 2}$ state.

This degenerate upper state with a similar angular configuration is also found in other atoms but with larger frequency separations than $\Delta \nu=1.1 \times 10^{10} \mathrm{~Hz}$ (Brasken \& Kyrola 1998) obtained for hydrogen. We will therefore adopt the terminology from those transitions: for $\mathrm{Ca}$ II, the transition from $J=1 / 2 \rightarrow J=3 / 2 \rightarrow J=1 / 2$ is denoted $\mathrm{K}$ (or $\mathrm{D}_{2}$ for $\mathrm{Na}$ I), while for the transition $J=1 / 2 \rightarrow J=1 / 2 \rightarrow J=1 / 2$, it is denoted $\mathrm{H}$ (or $\mathrm{D}_{1}$ for $\mathrm{NaI}$ ).

$H$ scattering: The wave function of the $2 P_{1 / 2}$ state has no angular dependence, and when it de-excites, conservation of momentum may result in a photon traveling in any direction, with any perpendicular polarization vector. Transitions through this state will yield a constant, angle-independent phase function and zero polarization independent of any prior polarization,

$$
p_{\mathrm{H}}\left(\theta^{\prime}, \phi^{\prime} \mid \rho_{\text {phot }}, \theta, \phi\right)=\mathrm{const},
$$

with the subsequent density matrix being $\left|c_{1}^{2^{\prime}}\right|=\left|c_{2}^{2^{\prime}}\right|=1 / 2$ and $c_{1}^{\prime} c_{2}^{\prime *}=c_{2}^{\prime} c_{1}^{*}=0$.

$K$ scattering: The wave function of the $2 P_{3 / 2}$ state, on the other hand, has a strong angular dependence. The phase function now depends on all the density matrix coefficients, and hence also on the incoming polarization. We present how the density matrix elements transform in Equations (19)-(21), as given in Equation (11) in Ahn \& Lee (2015). ${ }^{12}$ These transformations are given for a left-handed photon basis, with

\footnotetext{
${ }^{10}$ In the absence of circular polarization, $c_{1} c_{2}^{*}=c_{1}^{*} c_{2}$ if we have chosen a linear basis. This cross term then only gives the $U$ polarization. Otherwise, it also gives the $V$ polarization.

11 We use the notation $n L_{J}, n$ : energy level, $L=0,1,2,3, \ldots$ denoted $S, P, D$, $F, \ldots$ for the orbital angular momentum quantum number and $J=L+S$, where $S= \pm 1 / 2$ is the electron spin.

12 Or Equation (5) in Ahn et al. (2002).
}

one vector parallel to the plane of the scattering and the other perpendicular to it. The elements of the scattered density matrix obtained here are linear combinations of the elements of the incoming matrix, where the weights are determined by the incoming and scattered angles, as well as their differences.

Core scattering: We will from now on refer to the resonant $\mathrm{H}$ and $\mathrm{K}$ transitions collectively as core scatterings. The small frequency separation between the two makes it difficult to exactly determine the transition type. However, we use the fact that the effective ratio between the cross sections is $2 \lambda_{\mathrm{H}} / \lambda_{\mathrm{K}} \approx 2$ (Stenflo 1980). In the resonance core, $\mathrm{H}$ scattering is then twice as likely as $\mathrm{K}$ scattering.

\subsubsection{Wing Scattering}

As shown by Stenflo (1980), scattering far from the line center will, due to the interference between the two available sublevels of the excited Ly $\alpha$ state, resemble that of a classic oscillator. Wing scattering may be approached as a $J=0 \rightarrow J=1 \rightarrow J=0$ transition, which is the one representing Rayleigh and Thomson scattering alike (Chandrasekhar 1960). For this transition, we obtain the phase function and degree of polarization from the density matrix of Equation (4) in Ahn et al. (2002). Scattering at right angles yields 100\% polarization, while light that is forward- or backward-scattered retains its initial degree of polarization and the phase relation, thus preserving the polarization direction as well.

\subsection{Escape and Detection}

Detection is the last step involved in the Monte Carlo procedure. Observationally, the polarization properties of radiation are quantified by the Stokes parameters. To construct these parameters, we need to extract these from the polarization properties of individual photons in our Monte Carlo simulation (which is quantified by the density matrix/polarization state vector $\boldsymbol{P}$ ).

We achieve this by constructing a $3 \times 3$ "observable" density matrix $\rho_{\text {obs }}$ that projects the complex polarization state vector $\boldsymbol{P}$ (i.e., the density matrix; see Equation (2)) onto the plane of the sky defined by the observer. First, we specify the direction along which we "observe" our model. Without loss of generality, we define this direction to correspond to the $+z$ direction and thus assume that the plane of the sky corresponds to the $x y$ plane. We then only select those photons that escape within a solid angle $\omega$ from the $+z$ direction and calculate the Stokes parameters for each photon in this subset as follows:

$$
\begin{gathered}
I=\left|c_{x}^{2}\right|+\left|c_{y}^{2}\right|, \\
Q=\left|c_{x}^{2}\right|-\left|c_{y}^{2}\right|, \\
U=c_{x} c_{y}^{*}+c_{x}^{*} c_{y}=2 c_{x} c_{y}^{*}=2 c_{x}^{*} c_{y}, \\
V=i\left(c_{x} c_{y}^{*}-c_{x}^{*} c_{y}\right)=0,
\end{gathered}
$$

where the coefficients $\left|c_{x}^{2}\right|,\left|c_{y}^{2}\right|$, and $\left|c_{z}^{2}\right|$ and their phase relations $c_{x} c_{y}^{*}, c_{x} c_{z}^{*}$, and $c_{y} c_{z}^{*}$ and how these relate to the (intrinsic) density matrix of the photon, $\rho_{\text {phot }}$, are given in Appendix B. The last equalities of Equations (10) and (11) further indicate that we have no circular polarization, as we have neither emission of circularly polarized $\operatorname{Ly} \alpha$ nor processes that induce it.

We may then proceed to create images of the binned Stokes components, either for all frequencies or further binning the 
photons given their frequency. We may then define the degree of polarization,

$$
P=\frac{\sqrt{Q^{2}+U^{2}}}{I},
$$

and the relevant polarization angle,

$$
\chi=\frac{1}{2} \arctan \left(\frac{U}{Q}\right),
$$

in line with observational work (Hayes et al. 2011). The degree of polarization and polarization angle are thus derived quantities from the primarily binned Stokes parameters we calculated for each photon. Note also that $I^{2} \geqslant Q^{2}+U^{2}$ (see, e.g., Rybicki \& Lightman 1979), meaning that both $Q$ and $U$ may be zero when the intensity is not.

We have tested our implementation against known solutions. In Appendix C.1, we have tested our code against the scattering of a plane-parallel, semi-infinite slab known from Chandrasekhar (1960) for which Ahn \& Lee (2015) also obtained results. We have also tested against scattering in a Hubble-expanding cosmological volume known from Rybicki \& Loeb (1999) in Appendix C.2 and the expanding shell of Dijkstra \& Loeb (2008) in Appendix C.3. The density matrix implementation in tlac yields results equal to those of Ahn \& Lee (2015). Additionally, it reproduces the degree of polarization, as well as the surface brightness profiles for the expanding IGM and outflowing shell, even though the results that were compared to were obtained with the approach of Angel (1969), i.e., with fully polarized photons.

\subsection{Monte Carlo Implementation Summary}

We implement the density matrix formalism for polarization into tlac as follows.

1. We assign a $2 \times 2$ (possibly complex) density matrix $\rho_{\text {phot }}$ to each photon. We emit photons in a random direction $(\theta, \phi)$ and unpolarized. In practice, this means that we assign a density matrix with $\left|c_{1}^{2}\right|=\left|c_{2}^{2}\right|=1 / 2$ with no time-dependent correlation between them, i.e., $c_{1} c_{2}^{*}=c_{2} c_{1}^{*}=0$.

2. We generate an H I optical depth $\tau$ from the distribution $\exp (-\tau)$ and convert $\tau$ into a physical distance $s$ the Ly $\alpha$ photon travels before it escapes by solving the line integral $\tau=\int_{0}^{s} d r^{\prime} n_{\mathrm{HI}}\left(r^{\prime}\right) \sigma_{\alpha}\left(\nu\left[r^{\prime}\right]\right)$ (see Gronke \& Dijkstra 2014 for a more extended description of the code).

3. The new propagation direction after scattering depends on the phase function, which depends on the density matrix, which depends on the type of scattering event $(\mathrm{H}$ versus $\mathrm{K}$ versus wing) and the density matrix of the photon prior to scattering.

The frequency of the photon determines whether the scattering occurs in the damping wing or the core. For wing scattering, the postscattering density matrix is given by ${ }^{13}$ Equations (22)-(24). For core scattering, we draw a random number $\mathcal{R} \sim \operatorname{Unif}[0,1)$. If $\mathcal{R}>1 / 3$, the scattering is H-type, and the photon is depolarized $\left(\rho_{\text {phot }, 00}^{\prime}=\rho_{\text {phot }, 11}^{\prime}=1 / 2\right.$, other elements zero). Otherwise, the scattering is K-type, and the postscattering

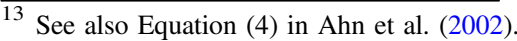

density matrix is given by ${ }^{14}$ Equations (19)-(21). We sample from these density matrices using the rejection method. We draw a random set of trial polar angles $\theta^{\prime}, \phi^{\prime}$ uniformly from a sphere and calculate the corresponding postdensity density matrix $\rho_{\text {phot' }}^{\prime}$,

$$
\rho_{\text {phot }}^{\prime}=f\left(\theta^{\prime}, \phi^{\prime} \mid \rho_{\text {phot }}, \theta, \phi\right),
$$

which in turn translates to the phase function, Equation (3). The phase function returns a number, which we compare to a randomly drawn number $\mathcal{R} \sim$ Unif $[0,1)$. If $\quad p\left(\theta^{\prime}, \phi^{\prime} \mid \rho_{\text {phot }}, \theta, \phi\right) \geqslant \mathcal{R}$, we accept the proposed scattering angles $\theta^{\prime}, \phi^{\prime}$, as well as the scattered density matrix $\rho_{\text {phot}}^{\prime}$, and the photon moves on

4. To create observable, well-defined Stoke parameters, the density matrix $\rho_{\text {phot }}$ can be transformed into a $3 \times 3$ density matrix $\rho_{\text {obs }}$ using Equations (29)-(37) that is relative to the observer, which is equivalent to observing the photons with a photon-counting device that is fixed in space and no longer oriented perpendicular to the propagation direction of the photon. This introduces six new density matrix coefficients: $\left|c_{x}^{2}\right|,\left|c_{y}^{2}\right|,\left|c_{z}^{2}\right|, c_{x} c_{y}^{*}=$ $c_{x}^{*} c_{y}, c_{x} c_{z}^{*}=c_{x}^{*} c_{z}, c_{y} c_{z}^{*}=c_{y}^{*} c_{z}$.

5. For a chosen coordinate axis (which one observes nadir), there will be a plane spanned by the other two coordinate vectors. For this plane, we obtain well-defined Stokes parameters $I, Q, U$, and $V$ (the latter is zero) through Equations (8)-(11).

6. The Stokes parameters of each photon can be binned (by, e.g., frequency, radial bins, and spatial pixels) to create observables for the chosen plane. Multiple planes can be combined by assuming symmetries to increase the signalto-noise ratio. We choose to observe photons escaping within a cone of $\cos 18^{\circ}=0.95$ of the axis-observed nadir, similar to Trebitsch et al. (2016), who chose $15^{\circ}$ and did not find the choice to strongly affect the results.

\section{Results}

Ly $\alpha$ radiative transfer through the interstellar and circumgalactic environments is a complex problem, and it is yet unclear which physical processes and scales play an important role in it. It is therefore advantageous to study Ly $\alpha$ radiative transfer in simplified geometries in order to better identify the precise origins of the predicted observables, that is, in our case, the predicted polarization signal. Here we present calculations of Ly $\alpha$ polarization for a suite of simplified systems that have been adopted in the literature. These are representative of features in more complex astrophysical systems. In particular, we will discuss

1. static (Section 3.1) or expanding (Section 3.2) ellipsoids,

2. biconical outflows (Section 3.3), and

3. clumps of HI clouds, representative of a multiphase medium (Section 3.4).

This can be thought of as a sequence in asymmetry. First, we introduce asymmetry in the gas distribution. Second, we add an asymmetry in velocity space. Third, we introduce further geometrical complexities by introducing biconical outflows. Fourth, we introduce "multiphase" versions of the outflow

\footnotetext{
${ }^{14}$ See also Equation (11) in Ahn \& Lee (2015)
} 

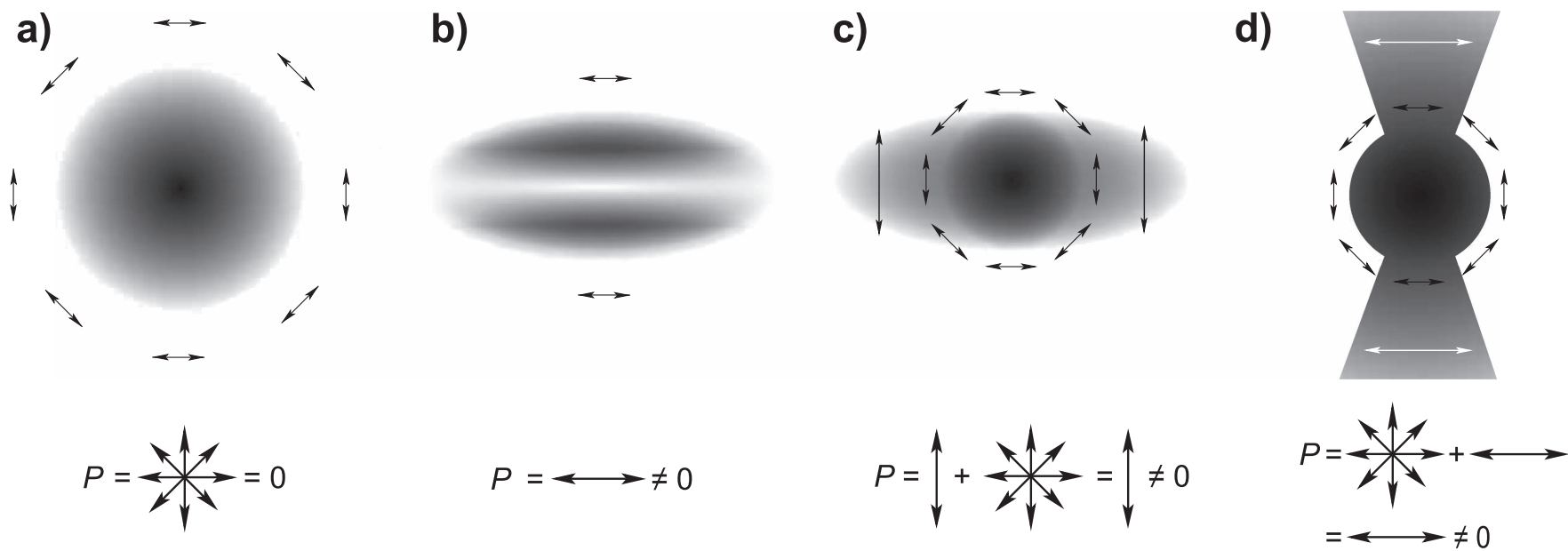

Figure 2. Sketches of four possible scattering geometries and their polarization signatures. (a) Spherically symmetric scattering geometry where the polarization increases toward the limb and is tangential to it. (b) Oblate ellipsoid where the majority of the intensity is polarized parallel to the plane of the major axes. (c) Optically thinner ellipsoidal scattering geometry where the majority of the intensity is polarized perpendicular to the major axes. (d) Bipolar outflow where the polarization is always perpendicular to the outflow axis and a symmetrically polarized unobscured central core. In the lower panels, we give the polarization if these extended sources were viewed edge-on as point sources, yielding (a) zero net polarization as all vectors cancel due to the circular symmetry of the extended polarization signal, where photons that escape further out do so by scattering increasingly closer to $90^{\circ}$, leaving in sum a polarization signal that increases radially and that always is oriented tangentially to the central source; (b) nonzero polarization for an ellipsoid, as the photons scatter and escape through optically thinner funnels along the minor axis of the ellipsoid before scattering at right angles toward the observer, becoming polarized horizontally; (c) nonzero polarization oriented perpendicular to the major axes of the ellipsoid, as the scattering geometry was thin enough to allow photons to scatter along the major axes and then toward the observer, with the only allowed polarization being in the vertical direction; and (d) nonzero polarization oriented perpendicular to the outflow axis, as all the contributions from the brighter core are canceled out. The shade indicates the intensity.

models. For each model, we will introduce the model parameters and present computed Ly $\alpha$ observables that could shed light on the nature and geometry of the sources and their environment. We sketch these models and some of our findings in Figure 2, which will be referred to throughout the text. Note that the apparent geometry of a system can change with frequency as Ly $\alpha$ photons of different frequencies escape at different spatial locations.

Following Lee \& Ahn (1998), we focus on computing the frequency dependence of polarization for point sources; this differs from more recent analyses, which focused on (frequency-)integrated properties of spatially extended sources (Dijkstra \& Loeb 2008; Dijkstra \& Kramer 2012; Trebitsch et al. 2016). This may represent a case where the Ly $\alpha$ source is spatially unresolved or a spectroscopic slit is wide enough to cover the entire source. For these point sources, we also show the total (i.e., integrated over frequency) polarization signal and its direction (relative to the unobservable geometry of the source in the plane of an observer).

\subsection{Oblate Ellipsoids}

Angel (1969) showed that Thomson scattering of thermal $\mathrm{X}$-rays emanating from an ellipsoidal scattering geometry could provide up to $5 \%$ polarization if it was viewed from the side as a point source. This was further explored by Kim et al. (2007), who considered $\mathrm{H} \alpha$ line and continuum radiation emanating from an ellipsoid that also acted as a Rayleigh scatterer. They found a viewing angle-dependent increase in polarization in the wings.

We pursue this idea further for Ly $\alpha$. We run a set of simulations where we explore the effects of changing (i) the column density of neutral $\mathrm{HI}$ gas and (ii) the ellipticity. Changing the column density is known to have a profound effect on the emergent spectrum. However, as shown in Dijkstra et al. (2016), the shape of the spectrum emanating from a source region fully enclosed in an ellipsoid will primarily be given by the axis of lowest column density, and therefore it cannot reveal the ellipticity.

We create an ellipsoid of static H I gas with fixed number density $n_{\mathrm{H} \mathrm{I}}$ with principal axes $\left(R_{\mathrm{ell}, a}, R_{\mathrm{ell}, b}, R_{\mathrm{ell}, c}\right)$, where we set the major axes $R_{\mathrm{ell}, a}=R_{\mathrm{ell}, b}$ and the perpendicular minor axis $R_{\mathrm{ell}, c}$. This ellipsoid embeds an inner ionized region with radius $R_{\mathrm{in}}$. In this inner region, we find our source of unpolarized Ly $\alpha$. The column densities along the principal axes can be found as $N_{\mathrm{H} \mathrm{I}}=n_{\mathrm{H} \mathrm{I}}\left(R_{\text {ell }}-R_{\text {in }}\right)$, i.e., the neutral hydrogen number density is constant throughout the system. The viewing angle $\mu$ is defined relative to the plane of the two major axes (see Figure 3 for a sketch of this geometry).

We choose three initial column densities along the minor axis: $N_{\mathrm{HI}}^{(c)}=10^{17}, 10^{19}$, and $10^{21} \mathrm{~cm}^{-2}$. The choice of column densities reflects those expected in real systems (Gronke et al. 2015; Hashimoto et al. 2017; Verhamme et al. 2017). The lower bound, $N_{\mathrm{HI}}^{(c)}=10^{17} \mathrm{~cm}^{-2}$, corresponds to a case from which ionizing LyC may escape. The upper bound, $N_{\mathrm{H} \mathrm{I}}^{(c)}=10^{21} \mathrm{~cm}^{-2}$, corresponds roughly to the upper envelope of $N_{\mathrm{H} \text { I }}$ that is inferred from Ly $\alpha$-emitting galaxies. We then vary the ellipticity by varying the major axes (i.e., $R_{\mathrm{ell}, a}$ and $R_{\mathrm{ell}, b}$ ). This gives a set of ellipticities $\varepsilon \equiv R_{\mathrm{ell}, c} / R_{\mathrm{ell}, a}=\{1,1 / 2,1 / 10,1 / 100\} .{ }^{15}$ We fix $R_{\text {in }}=10$ and $R_{\text {ell }, c}=20 \mathrm{pc}$ and note that the choice of scale is $\operatorname{arbitrary}^{16}$ for media that are static or have constant velocity fields. Our results are thus not scale-dependent. Furthermore, we set the gas temperate to $T=10^{4} \mathrm{~K}$ and inject the photons with $\sigma_{i}=200 \mathrm{~km} \mathrm{~s}^{-1}$.

In Figure 4(a), we plot the spectra of the intensity $I$ and the polarization $P$ for the emergent photons for $N_{\mathrm{H} \mathrm{I}}^{(c)}=10^{21} \mathrm{~cm}^{-2}$ and all ellipticities, viewed with $\mu=0$ (edge-on). As a guide to the eye, we plot (in this and other intensity spectra) a gray

\footnotetext{
15 A change in ellipticity is equivalent to changing the column density along the major axes, $N_{\mathrm{HI}}^{(a)}$.

${ }^{16}$ However, the choice of the ratio $R_{\mathrm{in}} / R_{\mathrm{ell}, c}$ may not be; we have, however, not investigated this further.
} 


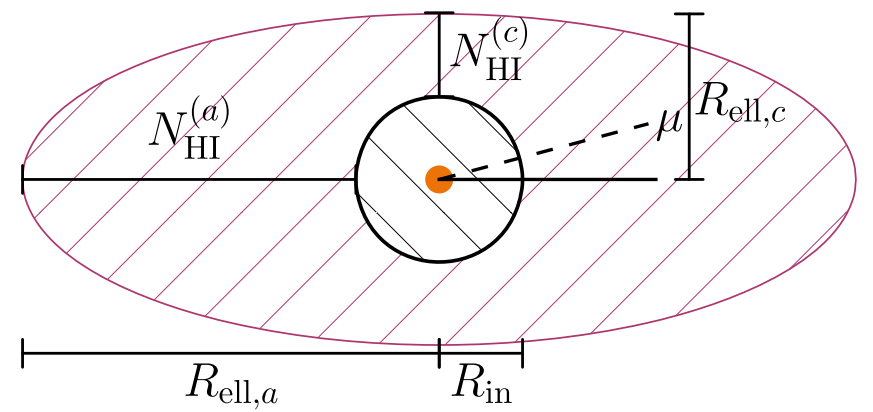

Figure 3. Ellipsoidal scattering geometry. We have an ionized inner region of radius $R_{\text {in }}$ where our Ly $\alpha$ source is located and an outer ellipsoidal H I region with principal axes $\left(R_{\mathrm{ell}, a}, R_{\mathrm{ell}, b}, R_{\mathrm{ell}, c}\right)$ where $R_{\mathrm{ell}, a}=R_{\mathrm{ell}, b}$. The outer ellipsoid has neutral hydrogen column densities $N_{\mathrm{H} \mathrm{I}}^{(a)}$ and $N_{\mathrm{HI}}^{(c)}$ along the principal axes. The viewing angle is given as $\mu$.

dashed line centered at $160 \mathrm{~km} \mathrm{~s}^{-1}$. This velocity offset marks a typical boundary between the redward, observable part of the spectrum and the blueward, inaccessible part, for which the increasingly neutral IGM at higher $z$ prevents transmission of Ly $\alpha$ photons (Dijkstra et al. 2007; Laursen et al. 2011).

As we are effectively changing the column density of the major axis, and the $(I)$ spectra do not change, we realize that their shapes are given by the column densities along the minor axis, which does not change, and not along the line of sight (LOS). In other words, the spectrum is mostly determined by the H I column density along the path of "least resistance" (see also Dijkstra et al. 2016 for a similar result). However, we find that $P$ is overall higher across the spectrum for increased ellipticities, including at those frequencies where most photons escape. This can be understood as a consequence of the increasing deformation of the source with increasing ellipticity. At all frequencies, the shape of the source becomes asymmetric, leaving a preferential polarization direction and an overall nonzero polarization signal.

We quantify this effect in Figure 4(b). Here we show the fractional degree of polarization $P$ as a function of (i) the minor axis column density $N_{\mathrm{H} \text { I }}^{(c)}$ and (ii) the ellipticity $\varepsilon$. We assume that we view the sources edge-on ${ }^{17}$ (i.e., the projected size of the source is $\left.R_{\mathrm{ell}, c} \times R_{\mathrm{ell}, a}\right)$. The color of a bin indicates the degree of polarization. The arrow indicates the direction of the linear polarization with regard to the plane of the major axes (the size of the arrow also reflects its magnitude).

The lower three panels of Figure 4(b) show that the degree of polarization is $P<1 \%$ for a spherical scattering geometry $(\varepsilon=1)$, as the spherical symmetry washes out any polarization. The polarization increases with ellipticity but in a way that depends nontrivially on $N_{\mathrm{HI}}^{(c)}$ : the direction of the polarization vector changes as $N_{\mathrm{HI}}^{(c)}$ increases from $N_{\mathrm{H} \mathrm{I}}^{(c)}=$ $10^{19}$ to $N_{\mathrm{H}}^{(c)}=10^{21} \mathrm{~cm}^{-2}$. This change in column density effectively blocks all light from the central part of the system, leaving only the photons that escape along the minor axes. See panel (b) of Figure 3 for a sketch of this obscuration. To reach the observer, they have to scatter closer to $90^{\circ}$, obtaining large degrees of polarization with the polarization vector oriented parallel to the major axes. This effect is fundamentally similar to the effect seen in spherically symmetric systems. At large radii, photons are also highly polarized, as they must escape these systems at $90^{\circ}$ to reach the observer; see, e.g., the rise in

\footnotetext{
$\overline{17}$ Face-on sources would appear circularly symmetric, and any polarization signal averages out.
}

$P$ with radius in Figure 3 of Dijkstra \& Loeb (2008). The polarization direction is always tangential to the central source. In these systems, the global signal would be canceled out from symmetries, however, as illustrated in panel (a) of Figure 2.

\subsection{Expanding Ellipsoids}

The previous section discussed the polarization emerging from static ellipsoids. Here we add an outflowing component

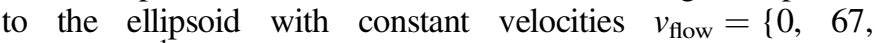
$200 \mathrm{~km} \mathrm{~s}^{-1}$, which is directed radially outward. As in Section 3.1, we fix $\sigma_{i}=200 \mathrm{~km} \mathrm{~s}^{-1}$. The presence of an outflow introduces an additional degree of asymmetry, now in velocity space.

Figure 5(a) shows the spectrum and frequency dependence of the polarization emerging from sources with fixed $N_{\mathrm{H} \text { I }}^{(c)}=10^{19} \mathrm{~cm}^{-2}$ along the minor axis, ellipticity $\varepsilon=1 / 10$, but with different expansion velocities $v_{\text {flow. }}$. For the static case, we recover the double-peaked spectrum that we obtained in Section 3.1. Expansion causes the majority of the photons to escape in the red wing, as blueward photons experience a higher optical depth (see, e.g., Zheng \& Miralda-Escudé 2002; Ahn et al. 2003; Dijkstra et al. 2006).

The frequency dependence of the polarization is also asymmetric around the line center. For the cases with $v_{\text {flow }} \neq 0$, there is little flux on the blue side of the line. The polarization of this flux is comparable to that of the static case (within the uncertainties). On the other hand, in the red wing of the line, outflows enhance the degree of linear polarization significantly. We obtain an increasing degree of linear polarization with velocity offset $\Delta v$ from the line center that approaches $P \sim 30 \%$ asymptotically at $\Delta v>500 \mathrm{~km} \mathrm{~s}^{-1}$. It is remarkable that the increase in the degree of linear polarization is very similar for all models with $v_{\text {flow }} \neq 0$. This result can be understood as follows. The distance a photon can travel increases for increasing outflow velocities, effectively lowering the optical depth seen by the photons. In our cases, the change in outflow velocities would not necessarily imply a change in the observed spatial shape of the system. The optical depth is sufficiently low to make a significant fraction of the photons diffuse along the major axes in the presence of outflows, with polarization vectors tangential to the direction of the central source, as sketched in panel (c) of Figure 2. This means that the spatial asymmetry does not change significantly, and the degree of polarization remains similar between the models.

Figure 5(b) shows the degree and direction of polarization of expanding ellipsoids with $N_{\mathrm{H}}^{(c)}=\left\{10^{19}, 10^{21}\right\} \mathrm{cm}^{-2}$ and $\varepsilon=1 / 10$ viewed edge-on. We omit the case $N_{\mathrm{H} \mathrm{I}}^{(c)}=10^{17} \mathrm{~cm}^{-2}$ because we found that for this case, too few photons scatter, and our predictions practically correspond to that assumed for the intrinsic source. For $N_{\mathrm{HI}}^{(c)}=10^{19} \mathrm{~cm}^{-2}$, the polarization is near zero for the static ellipsoid (in agreement with the upper middle panel of Figure 4(b)). When we increase $v_{\text {flow }}$, the polarization remains roughly constant $P \sim 10 \%$, with the polarization direction aligned perpendicular to the major axes. This can be understood to be for the same reasons that the degree of polarization in the spectra did not change. The spatial (observed) shapes of the systems do not significantly change for the increasing outflow velocities. As the asymmetries do not change, the systems obtain similar degrees and directions of polarization.

Figure 5(b) contains other interesting results. For a higher column density along the minor axis, $N_{\mathrm{HI}}^{(c)}=10^{21} \mathrm{~cm}^{-2}$, the polarization behaves completely differently compared to the case $N_{\mathrm{H}}^{(c)}=10^{19} \mathrm{~cm}^{-2}$. The total polarization of $P=8 \%$ for a static 


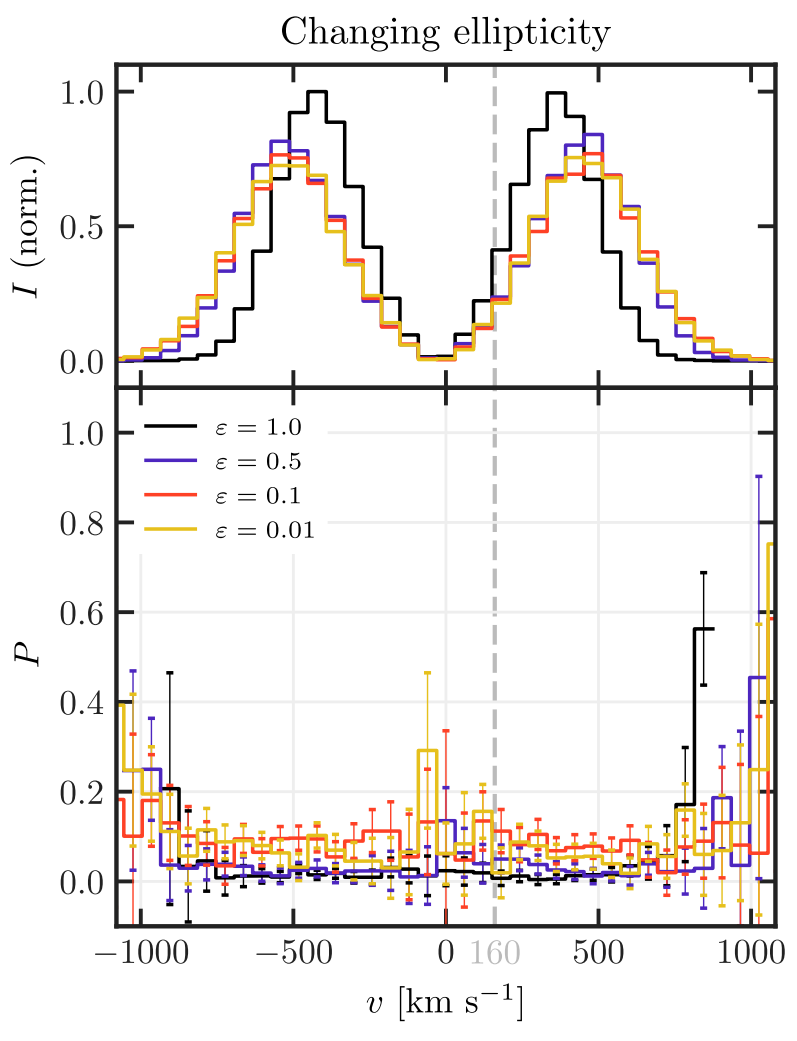

(a)

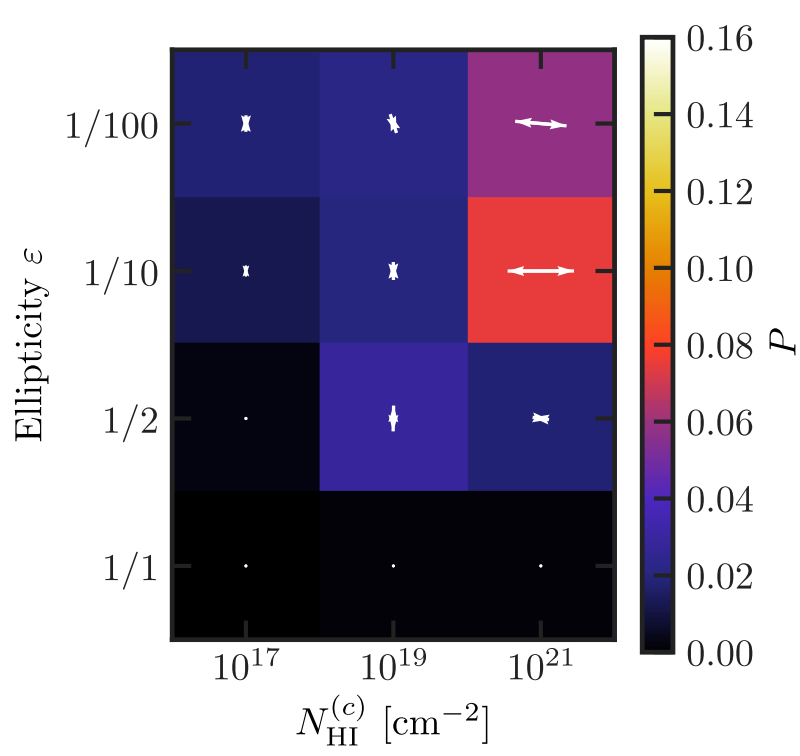

(b)

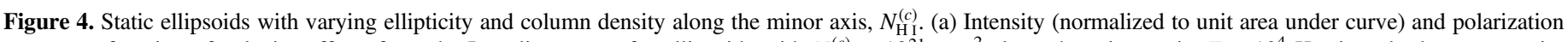

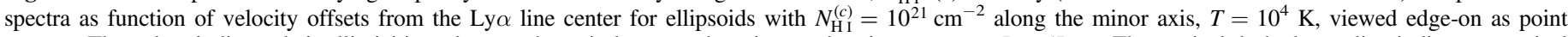

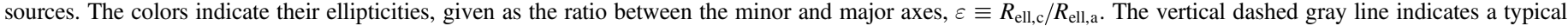

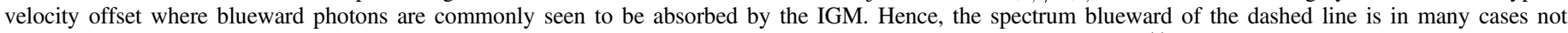

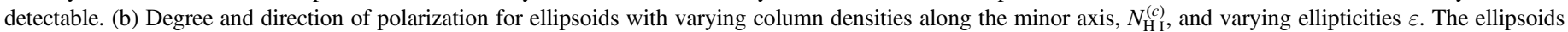

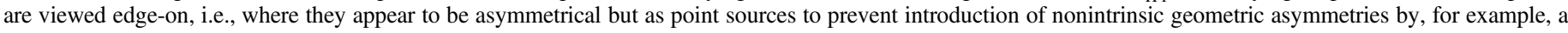

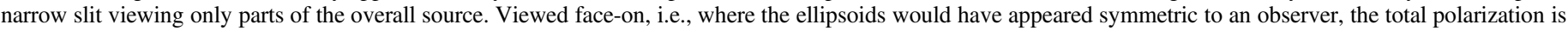

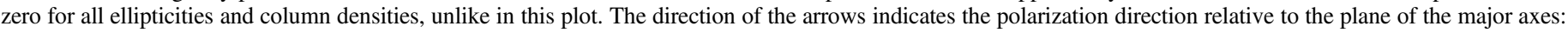
horizontal arrows are parallel to it, whereas vertical arrows are perpendicular to it.

ellipsoid (see also Figure 4(b)), and it now decreases with outflow velocity, reaching $P=1 \%$ for $v_{\text {flow }}=200 \mathrm{~km} \mathrm{~s}^{-1}$. For this higher column density, the system is seen transitioning from the state sketched in panel (b) in Figure 2 to the more symmetric case presented in panel (c). However, there is one important difference from the system presented in panel (c), as well as the system with lower column density $N_{\mathrm{HI}}^{(c)}$. The increase in optical depth from the increased column density means that the photons also increasingly scatter once they encounter the rest-frame velocity offset of the atoms in the expanding medium. This isotropizes the local radiation field, and the polarization vectors become randomized. The lowering in optical depth from the increasing outflow means the photons penetrate deeper also along the major axes, contributing to removing the spatial asymmetry. The overall effect is that the net polarization is reduced. This reduction is eventually accompanied by a flip in the polarization vector of the $N_{H i}^{(c)}=10^{21} \mathrm{~cm}^{-2}$ system, where the vector changes alignment from being parallel to perpendicular to the major axes. This indicates an increased semblance to the geometry of the much lower column density system $N_{\mathrm{H} \text { I }}^{(c)}=10^{19} \mathrm{~cm}^{-2}$.

\subsection{Bipolar Outflows}

So far, our analysis has focused on spherically or cylindrically symmetric gas geometries. However, there is observational and theoretical evidence that outflows are bipolar (e.g., Blandford \& Rees 1974; Suchkov et al. 1994). More recently, observations of LARS 05 (Duval et al. 2016) nicely illustrate how Ly $\alpha$ photons scatter off a bipolar outflow that burst out of an edge-on disk galaxy. In this section, we focus on predicting spectra and polarization of scattered Ly $\alpha$ radiation emerging from simplified representations of bipolar outflows, with either an unobscured (Section 3.3.1) or obscured (Section 3.3.2) central source.

We model the bipolar outflow as follows. It contains a spherical cloud with $N_{\mathrm{H}}=\left\{10^{17}, 10^{19}, 10^{21} \mathrm{~cm}^{-2}\right.$ and $T=10^{4} \mathrm{~K}$ that resides in a fully ionized environment. The (unpolarized) Ly $\alpha$ source resides in the center of this cloud. We then introduce bipolar outflows in cones with total opening angles $\theta_{\text {flow }}=\{1 / 16$, $1 / 8,1 / 4,1 / 2\} \pi$. Inside the cones, gas is radially outflowing with a constant velocity $v_{\text {flow }}=200 \mathrm{~km} \mathrm{~s}^{-1}$. The H I number density in the cones is equal to that in the central sphere and extends a factor of 4 further than the edge of the sphere.

\subsubsection{Unobscured Central Source}

Figure 6 shows an illustrative example of the spatial distribution of intensity and polarization for an outflow with $\theta_{\text {flow }}=\pi / 8$ viewed edge-on. ${ }^{18}$ We clearly see the biconical

\footnotetext{
${ }^{18}$ When viewing the outflows face-on, i.e., straight into the cones and possibly also the central static cloud, one would observe a spherically symmetric source, and hence any point-source polarization signal would be lost.
} 


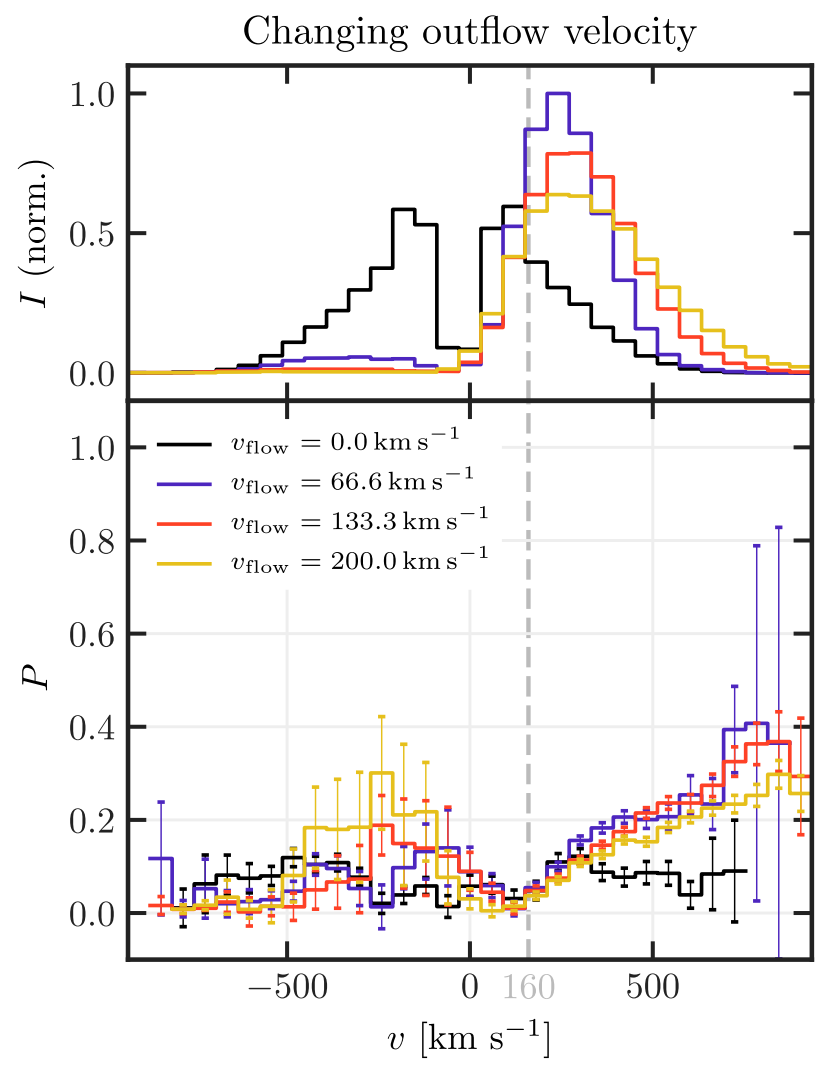

(a)

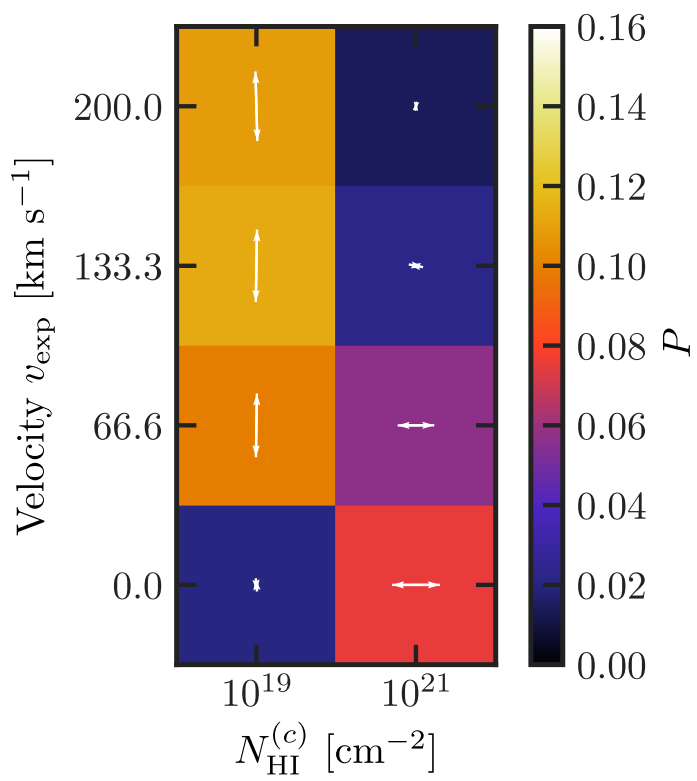

(b)

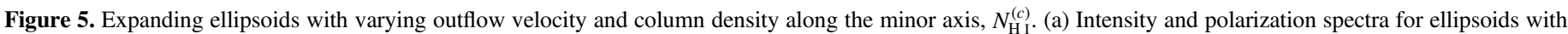

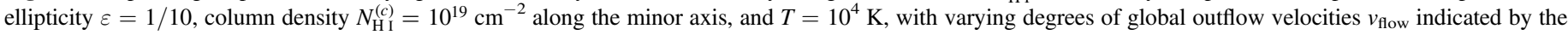

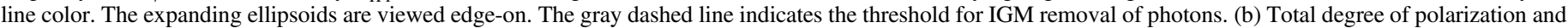

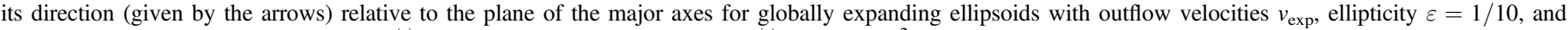
column densities along the minor axis $N_{\mathrm{HI}}^{(c)}$. The spectra of the sources with $N_{\mathrm{HI}}^{(c)}=10^{19} \mathrm{~cm}^{-2}$ are plotted in Figure $5(\mathrm{a})$.

structure in both the intensity and the polarization images. In the central, static sphere, the degree of polarization increases familiarly toward the limb, reaching $P \sim 30 \%$ with the polarization angle oriented tangentially to the center. The intensity decreases radially outward, both in the central cloud and in the cones. This diffusion of photons gives rise to a decreasing surface brightness profile; see, e.g., Figure 4 of Dijkstra et al. (2006). In the outflows, the degree of polarization increases with $\Delta v$, reaching values of $P \sim 50 \%-70 \%$ (pixels with $P>80 \%$ exist, but the flux in these is negligible). The direction of polarization in the outflows is oriented perpendicular to the flow direction.

Figure 6 also shows that the overall degree of polarization signal increases with the opening angle $\theta_{\text {flow }}$ and is always aligned perpendicular to the outflow direction. This is the same physical effect that we saw at play for the ellipsoids: the local radiation fields are stronger in the direction of the source and not isotropized, leaving a polarization vector tangential to the direction of the central source.

The dependence of integrated polarization on $\theta_{\text {flow }}$ is summarized in Figure 7, which shows clearly that $P$ increases with $\theta_{\text {flow }}$ for all $N_{\mathrm{H} \text { I }}^{(c)}$. This increase reflects that an increasing $\theta_{\text {flow }}$ causes a larger fraction of flux to emerge from the biconical outflows, thereby increasing the spatial asymmetry of the source. Additionally, the biconical outflows appear more polarized: fewer photons scatter here. Those that do propagate along the outflow direction but have to scatter and escape at
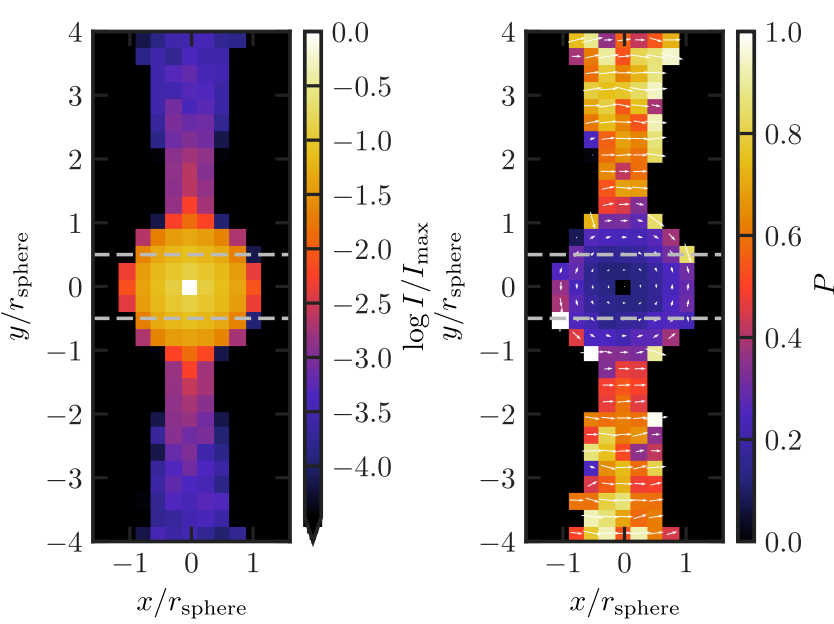

Figure 6. Spatially extended maps of the intensity and degree of polarization for bipolar outflows of $v_{\text {flow }}=200 \mathrm{~km} \mathrm{~s}^{-1}$ from a static sphere with $N_{\mathrm{H} \mathrm{I}}=10^{19} \mathrm{~cm}^{-2}$ and a total opening angle of $\theta_{\text {flow }}=\pi / 8$. The colors indicate the degree of polarization, and the arrows indicate the direction of polarization. The dashed lines indicate the region that is obscured in Figure 8 to resemble the removal of Ly $\alpha$ photons by, for example, a dusty disk.

angles closer to $90^{\circ}$ to reach the observer. That way, they gain large degrees of polarization that also are oriented perpendicular to the outflow axis. We illustrate this in panel (d) of Figure 2. 


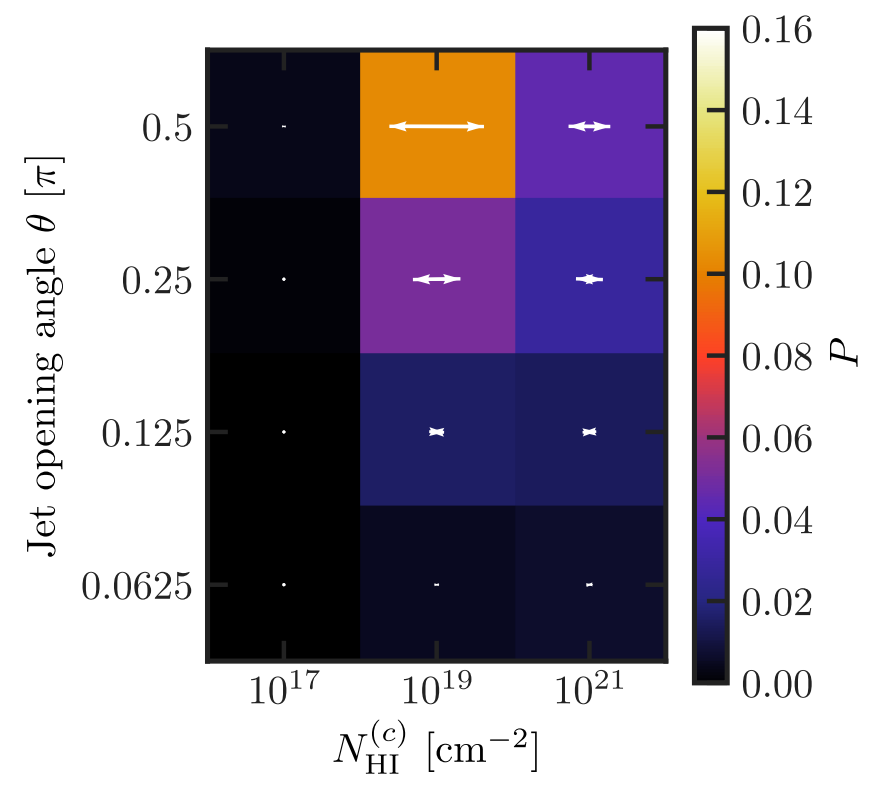

Figure 7. Degrees of polarization had the unobscured bipolar flows (with outflow velocity $v_{\text {flow }}=200 \mathrm{~km} \mathrm{~s}^{-1}$ ) been viewed as a point source. The direction of the overlaid polarization vectors indicates their direction relative to the flow (horizontal: perpendicular to flow directions; vertical: parallel to flow directions).

Figure 7 also shows that the overall polarization is maximal for $N_{\mathrm{H}}=10^{19} \mathrm{~cm}^{-2}$. For $N_{\mathrm{H} \mathrm{I}}=10^{17} \mathrm{~cm}^{-2}$, the central cloud is optically thin to most emitted Ly $\alpha$ photons, and there is little flux in the scattered component (and the flux that does scatter, scatters in the core, which leads to a lower degree of polarization). The overall polarization for $N_{\mathrm{H} \mathrm{I}}=10^{21} \mathrm{~cm}^{-2}$ is lower because in this case, the scattering medium is optically thicker, which isotropizes the $\operatorname{Ly} \alpha$ radiation field. In turn, this isotropization reduces the overall polarization of the radiation that escapes.

The upper left panel of Figure 8 shows the spectra of the models with $N_{\mathrm{H} \mathrm{I}}=10^{19} \mathrm{~cm}^{-2}$ (i.e., with maximum polarization). The spectra are double-peaked, with the red peak becoming stronger relative to the blue peak with increasing $\theta_{\text {flow. }}$. This increase reflects the increasing fraction of Ly $\alpha$ photons that scatter through the outflow. The lower left panel of Figure 8 shows that the degree of polarization is negligible blueward of line center for all opening angles. Redward, the degree of linear polarization increases with $\Delta v$. This reflects that the blue peak consists of photons that escape from the static central cloud: polarization is canceled out from the symmetric central geometry (as for case $a$ in Figure 2). This also holds to some extent for the red peak, but, in addition, it has contributions from photons that have escaped into the biconical outflows. There, they scatter less and provide a higher overall local polarization signal. The redward increase in the global polarization reflects the increasing spatial asymmetry with $\Delta v$.

\subsubsection{Obscured Central Source}

We repeat the previous analysis (Section 3.3.1) but now obscure the central static sphere (the obscured region indicated with dashed gray lines in Figure 6). This represents a case in which the biconical outflows are separated by, for example, a dusty galactic disk, as in LARS 05 (see Duval et al. 2016) or M82 (Lynds \& Sandage 1963; Gallagher \& Smith 1999).

The right panels of Figure 8 show the spectra and polarization for the same models as in the left panels but with the central region obscured. Especially the red peaks of the spectra are widened for the largest opening angles $\theta_{\text {flow }}=\{1 / 4,1 / 2\} \pi$. This enhancement of the red peak is primarily a renormalization of the entire spectrum. The obscuration removes a majority of the (blue and red) photons that arise from the central spherical cloud, leaving the surplus of red photons that escape from the cones. For smaller opening angles $\theta_{\text {flow }}$, however, less of the overall flux originates from the bipolar cones. The surplus of red photons that was seen for the larger opening angles is present but is, however, not sufficient to significantly alter the shape of the spectrum. The spectral signature of the outskirts of the central sphere therefore dominates the spectrum.

The lower right panel shows that the polarization increases at effectively all frequencies. This simply reflects that obscuring the central source eliminates photons whose polarization vectors align with the cone axis. The polarization in the blue wing is lower than that in the red wing, as these are photons that escape from primarily the central sphere. The additional boost in $P$ at large $\Delta v$ in the red wing is the signature of the photons that have scattered in the outflows. As for the unobscured case, the local polarization is higher due to the fewer scatterings photons here undergo, and the global degree of polarization reflects the spatial asymmetry due to the cones. The degree of polarization in the red wing reveals how the source transitions from nearly symmetrical, without much contribution from the cones for $\theta_{\text {flow }}=1 / 16$, to larger contributions from the cones with increasing $\theta_{\text {flow }}$. This comes at a price: the increased opening angles also allow for larger variations in the polarization vectors, which, in the cones, are tangential to the source.

\subsection{Multiphase Medium}

All previous models represented gas in the ISM with a single density and temperature. In reality, interstellar (and circumgalactic) gas is known to be multiphase. $\operatorname{Ly} \alpha$ radiative transfer through multiphase media is a complex problem, which has also been represented by simplified models (see, e.g., Neufeld 1991; Hansen \& Peng Oh 2006; Dijkstra \& Kramer 2012; Laursen et al. 2013; Gronke \& Dijkstra 2016). These simplified models consist of neutral, spherical (possibly dusty) clumps embedded within a hot, ionized, and dust-free medium (based loosely on the early models by McKee \& Ostriker 1977).

It has been demonstrated that for such "clumpy" media, the key parameter that affects $\mathrm{Ly} \alpha$ radiative transfer is the average number of clumps per sightline: the covering factor $f_{\mathrm{c}}$ (see Hansen \& Peng Oh 2006; Gronke \& Dijkstra 2016). Gronke \& Dijkstra (2016) and Gronke et al. (2017) showed that there exists a critical value for $f_{\mathrm{c}}, f_{\mathrm{c} \text {,crit }}$, above which clumpy media affect Ly $\alpha$ photons as if they consist of a single phase (i.e., homogeneous). The value of $f_{\mathrm{c}, \text { crit }} \sim$ a few to a few tens, depending on the total $\mathrm{H}$ I column density and kinematics of the clumps (see Gronke et al. 2017). The polarization properties of Ly $\alpha$ radiation that scatters through "very clumpy" media (i.e., $f_{\mathrm{c}} \gg f_{\mathrm{c} \text {,crit }}$ ) are therefore well captured by our previous models, in which the gas was homogeneously distributed. The 

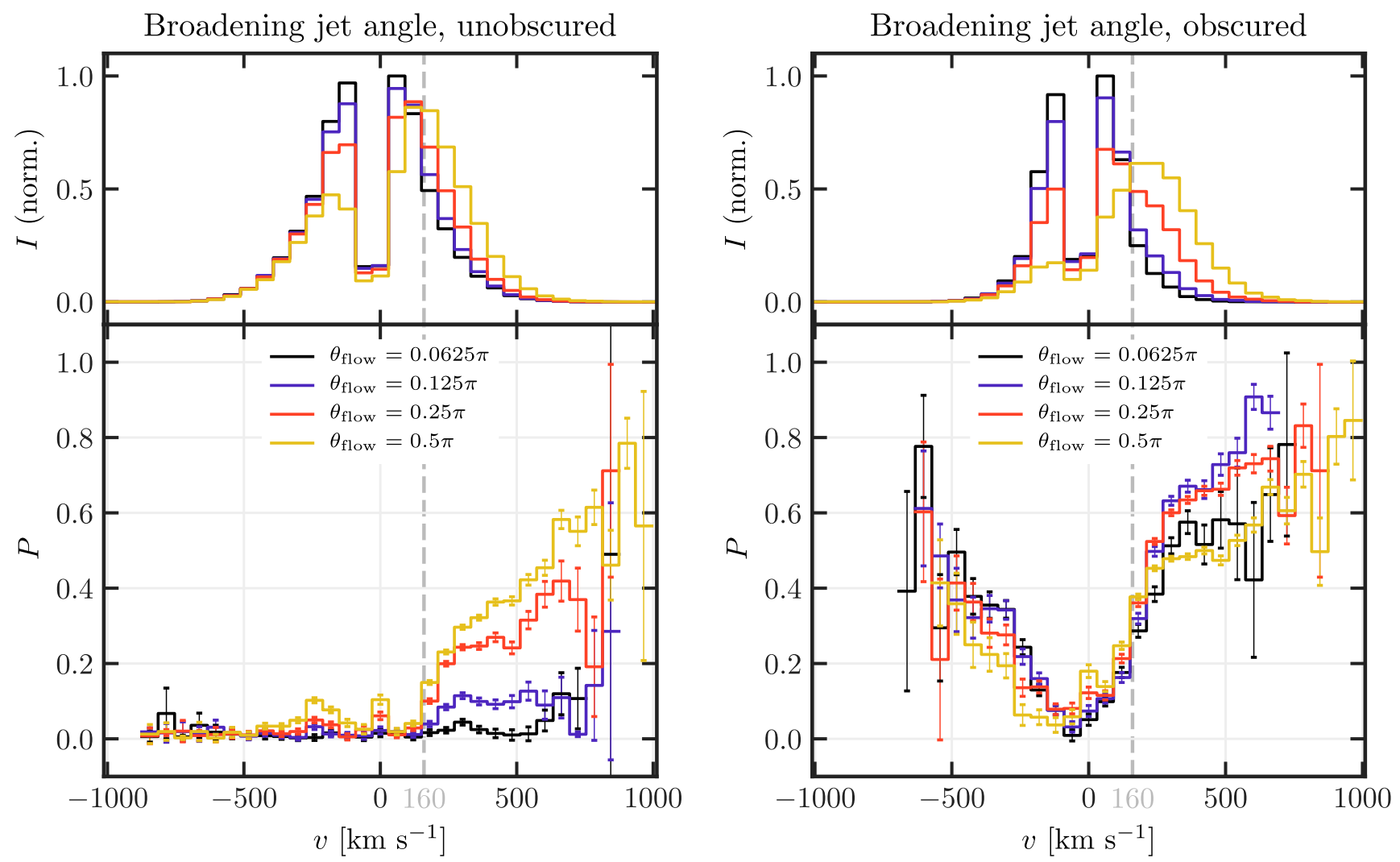

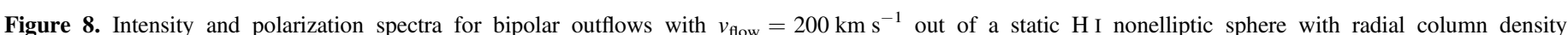

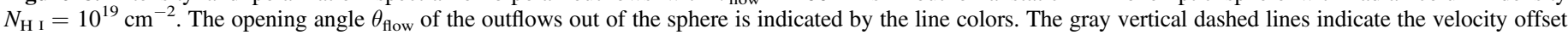

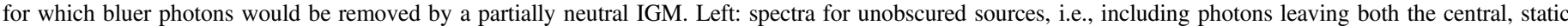

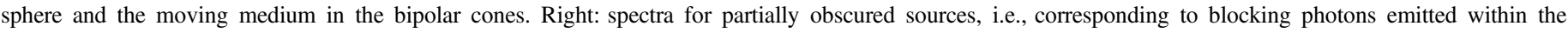
horizontally dashed gray lines in Figure 6, resembling, for example, the removal of Ly $\alpha$ photons by a circumgalactic disk of dust.

polarization properties of $\operatorname{Ly} \alpha$ radiation through models with $f_{\mathrm{c}} \ll f_{\text {c,crit }}$ have been explored in Dijkstra \& Kramer (2012), where it was associated with few (or no) scatterings and consequently high degrees of polarization.

In this section, we focus on the "transition regime," which corresponds to $f_{\mathrm{c}} \sim f_{\mathrm{c} \text {,crit }}$, and contrast a central Ly $\alpha$-emitting source surrounded by a uniform distribution of randomly moving clumps (which can represent a central star-forming galaxy surrounded by a clumpy circumgalactic medium) with a setup where the Ly $\alpha$ radiation emerges from the clumps (which can represent the same galaxy and circumgalactic medium but in which Ly $\alpha$ arises as fluorescent emission powered by ionizing radiation that leaked from the central galaxy; see MasRibas \& Dijkstra 2016; Mas-Ribas et al. 2017). While the numerical value of $f_{\text {c,crit }}$ depends on the neutral hydrogen column density of the clumps $N_{\mathrm{H} \mathrm{I,cl}}$ and their kinematics (Gronke et al. 2017), we stress that the characteristics described in this section generally apply for systems with $f_{\mathrm{c}} \sim f_{\mathrm{c}, \text { crit }}$.

In our models, we chose the clumps' column densities to be $N_{\mathrm{H} \mathrm{I}, \mathrm{cl}}=10^{18} \mathrm{~cm}^{-2}$ with a gas temperature of $T=10^{4} \mathrm{~K}$ (motivated by the "shattering" theory of McCourt et al. 2016) and a random velocity with each component drawn from a Gaussian distribution with standard deviation $\sigma_{\mathrm{cl}}=200 \mathrm{~km} \mathrm{~s}^{-1}$. This yields a critical covering factor of $f_{\mathrm{c}, \text { crit }} \approx 5$ (Gronke et al. 2017), which we will adopt for $f_{\mathrm{c}}$. Furthermore, we choose the clumps' radii to be $r_{\mathrm{cl}}=1 \mathrm{pc}$ and fix the radius of the (spherical) system to $1 \mathrm{kpc}$. We note, however, that these parameters (given that the others are fixed) do not influence the radiative transfer process (Hansen \& Peng Oh 2006). We set the intrinsic spectrum to have $\sigma_{i}=12.85 \mathrm{~km} \mathrm{~s}^{-1}$ (in the reference frame of the emitting gas), which corresponds to the thermal velocity of the gas.

The upper left panel of Figure 9 shows the spectra from a multiphase medium where Ly $\alpha$ is emitted either (a) by a central source or (b) extendedly, throughout the medium by sources residing in each clump. This is particularly visible in the intrinsic spectra, plotted with dashed and dotted lines. With the central source, the photons are emitted close to the line center. With the extended source, the motion of the clumps must also be accounted for. Both spectra are broad and single-peaked, which is characteristic of media with $f_{\mathrm{c}} \sim f_{\mathrm{c} \text {, crit }}$ (see Figure 3 of Gronke et al. 2017). Both models also predict a degree of spatially averaged polarization that is consistent with zero over most frequencies. This is a direct consequence of our symmetric scattering geometry-even if there exist patches that are tangentially polarized to the center, the overall geometry will cancel the global signal out, as illustrated in Figure 2. The apparent rise in polarization toward the far wings occurs at frequencies with near-zero intensity.

In the right panel of Figure 9, we plot the normalized surface brightness profiles (which we after normalization render unitless by dividing with $\mathrm{I}_{\max }$, the maximum surface brightness of the model with a central source) and polarization profiles of the sources as function of impact parameter $\alpha$ in $\mathrm{kpc}$, in 

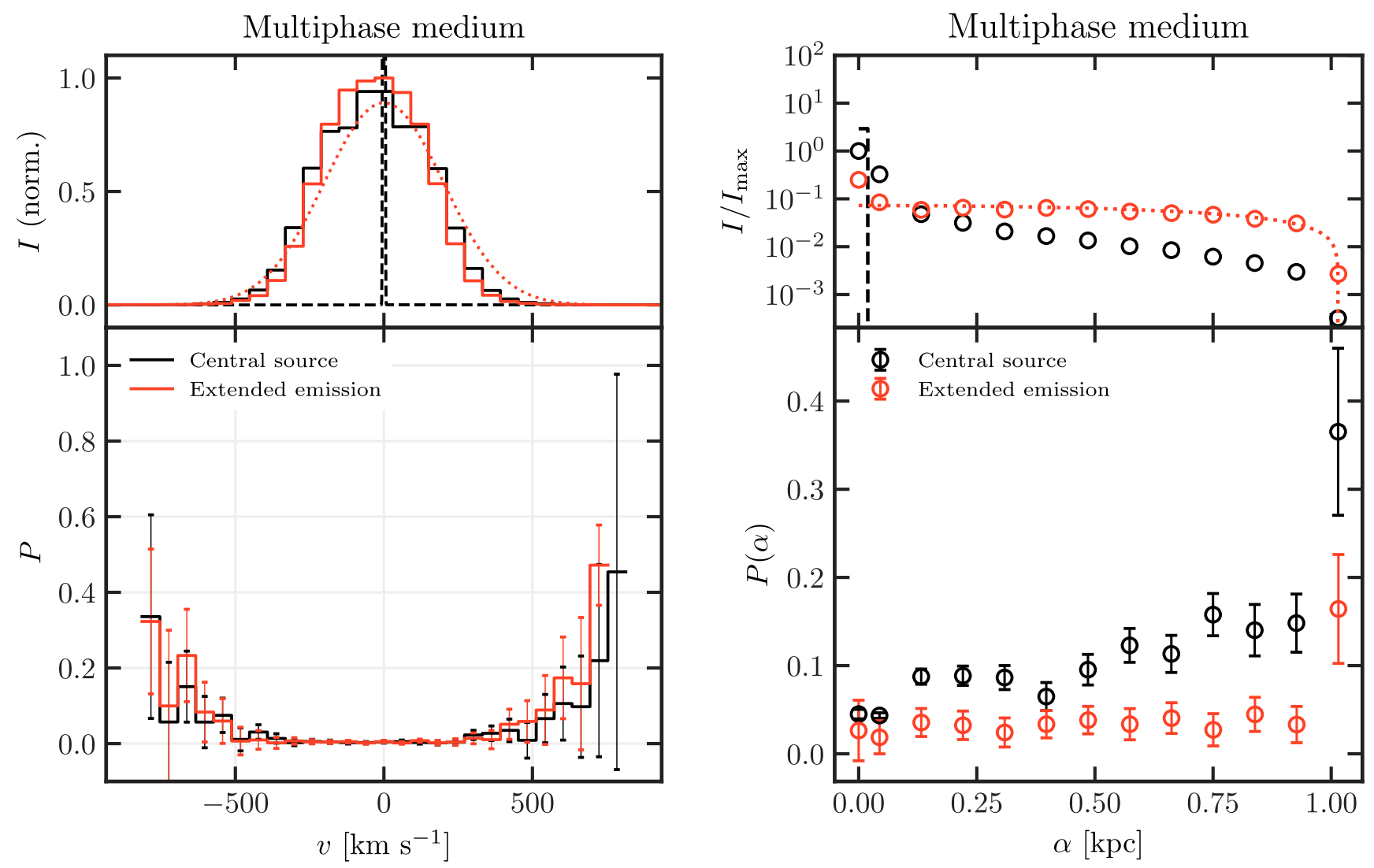

Figure 9. Left: spectra of intensity and degree of polarization as a function of velocity offset/frequency from the Ly $\alpha$ line center for systems filled with many small clumps of $\mathrm{H}$ I gas, sized to provide a covering fraction $f_{\mathrm{c}} \sim f_{\mathrm{c} \text {, crit }}$, in an otherwise ionized medium, representative of a multiphase scattering system. We plot the emergent spectra for systems where the source of Ly $\alpha$ is central (black line) or extended throughout the medium (red line), i.e., embedded in each cloud. Note that we do not view the sources through a slit, but rather as point sources. We also plot the intrinsic intensity spectra; the dashed black line is for the central source, and the red dotted line is for the extended source. Right: surface brightness (normalized and rescaled to the maximum value obtained in the two models) and polarization profiles as a function of impact parameter $\alpha$ for a central (black) and extended (red) source of Ly $\alpha$ emission in a clumpy, multiphase medium. We also plot the intrinsic surface brightness profiles; the dashed black line is for the central source, and the red dotted line is for the extended source.

agreement with previous studies (see Dijkstra et al. 2006). We also plot the intrinsic, unscattered surface brightness profiles with dotted/dashed lines. In addition, the degree of polarization also differs. For the central source, $P$ rises to $\sim 10 \%$ at $\sim 0.1 \mathrm{kpc}$ before it eventually rises to $\sim 15 \%$. For extended sources of Ly $\alpha$ emission, the polarization is consistent with $P<5 \%$ out to the most distant impact parameters. The degree of polarization is lower than that obtained for scattering off clumps at low $f_{c}<1$ and greater $N_{\mathrm{H} \mathrm{I}, \mathrm{cl}}$ in Dijkstra \& Kramer (2012), where photons that scattered only once in a clumpy outflow would produce a spatial polarization signal up to $P \approx 60 \%$ at large impact radii. As we have a higher number of clumps along the LOS (and thereby a higher total optical depth) and a lower clump optical depth, the photons scatter several times per clump, which reduces their polarization. This explains the lower $P$ we obtain in the case of a central source as compared to Dijkstra \& Kramer (2012). However, we still obtain a similar increase in $P$ with impact radii, as in other models with a central source, since the radiation field is anisotropic, being stronger in the direction of the source, and the photons that escape at large radii must do so by scattering increasingly more at $90^{\circ}$.

This means, even though the exact degree of polarization depends on other parameters, such as $N_{\mathrm{H} \text { I,cl }}$ and the clump placement, the central source shows a rising $P(r)$ signal, while the "fluorescent" clumps (i.e., the extended source) do not. This is a clear observational signal for the distinction of different Ly $\alpha$-powering mechanisms, and we will explore this further in future work.

\section{Discussion}

In this section, we discuss the origins of polarization from quantum mechanical (Section 4.1) to astrophysical scales (Section 4.2). We discuss how $\mathrm{Ly} \alpha$ polarization can break degeneracies between models for spectra and/or surface brightness profiles when used in concert with these other observables (Section 4.3).

\subsection{Polarization: The Quantum Mechanical Origins}

For classical electron scattering - which applies to Ly $\alpha$ wing scattering-unpolarized radiation that scatters at right angles becomes maximally polarized. In addition, the polarization properties of a single photon impose restrictions on the scattering angles: the photon cannot scatter in the direction in which it is fully polarized. For core scattering, however, the "shape" of the quantum mechanical wave functions plays a role. Unpolarized radiation still obtains the highest degree of 
Table 1

Polarization through Single Scatterings

\begin{tabular}{lccccc}
\hline \hline \multirow{2}{*}{ Init. Polarization } & \multicolumn{2}{c}{ Core } & & \multicolumn{2}{c}{ Wing } \\
\cline { 2 - 5 } \cline { 5 - 6 } & $90^{\circ}$ & $0 / 180^{\circ}$ & & $90^{\circ}$ & $0 / 180^{\circ}$ \\
\hline Unpolarized $^{\mathrm{a}}$ & $43 \%$ & $0 \%$ & $100 \%$ & \\
Polarized $^{\mathrm{b}}$ & & & & Unchanged \\
\hline
\end{tabular}

Note. Core scattering in this regard includes only the anisotropic K transition, not the depolarizing $\mathrm{H}$ transition.

${ }^{a}$ An initially unpolarized photon has $P=0 \%$.

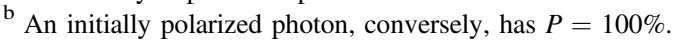

polarization when scattering at right angles, but only up to $P=43 \%$ via $\mathrm{K}$ scatterings (i.e., through the $2 \mathrm{P}_{3 / 2}$ state). For $\mathrm{H}$ scatterings (i.e., through the $2 \mathrm{P}_{1 / 2}$ state), polarization is only destroyed as the wave function of the $2 \mathrm{P}_{1 / 2}$ is spherically symmetric. For fully polarized radiation, i.e., with $P=100 \%$, a photon that is $\mathrm{H}$-scattered will only obtain $P=43 \%$ for scattering at right angles, but for forward or backward scatterings, the degree of polarization can increase to $P=$ $60 \%$. This effect is known as depolarization: a photon can only obtain $P=100 \%$ through wing scatterings, and if it is scattered through the core, it will only retain, at best, $P=60 \%$. Depolarization is not possible through wing scatterings; a partially polarized photon that is forward- or backward-scattered will retain its polarization or have it boosted when scattering at inclined angles.

We obtain these polarization magnitudes by using the desired scattering angles for the density matrices of the various transitions. These values agree perfectly with the results for Rayleigh or core scatterings as described in, e.g., Chandrasekhar (1960) or Dijkstra \& Loeb (2008). We summarize this discussion in Table 1, which provides an overview of the polarization obtained through single scatterings.

\subsection{Polarization: The Astrophysical Origins}

There is a difference between the probability of measuring the polarization state of an individual photon-which we have just shown can grow to prefer highly polarized photons after multiple scatterings-and observationally detecting polarization. The observable Stokes parameters describe the polarization properties of an ensemble of photons. While individual photons may obtain high levels of linear polarization through scattering, the ensemble-averaged polarization can still be zero if the polarization angles are not well aligned.

There are two main mechanisms behind the alignment of the polarization vectors, and thus, of the generation of observable polarization: natural asymmetries in the scattering geometry and introduced asymmetries from, e.g., finite slit widths in spectropolarimeters, foregrounds, or instrumental artifacts. We discuss these next.

I. Natural asymmetries. On scales where the properties of the scattering medium appear constant (local scales), any process that induces some preference in scattering direction also introduces a preferential polarization direction. The polarization cannot be oriented in the direction the photon had before scattering, and it must be perpendicular to the postscattering propagation direction. Such a process can be an alignment of the atoms in the medium either from an external magnetic field or from pumping by scatterings (see, e.g., Zhang \& Yan 2018) or, as in our cases, an anisotropic radiation field, as also realized by Dijkstra \& Loeb (2008). A small, local patch of the scattering medium is, in general, unevenly illuminated, with the strongest illumination in the direction of the source. This is the origin of the tangential polarization patterns one would obtain from scatterings in the expanding IGM (Rybicki \& Loeb 1999), spherical shells (Dijkstra \& Loeb 2008), or any of the symmetric regions of our scattering models, including the spherical ellipsoid $(\varepsilon=1 / 1)$ in Section 3.1, the centrally illuminated clumpy medium in Section 3.4 with the radial polarization profile given in Figure 8, or the IGM (radial profile in Figure 12). Such a polarization pattern of concentric circles was observed in LAB1 by Hayes et al. (2011; illustrated in Bower 2011). At larger impact radii, these models possess a larger degree of polarization. This is due to the larger fraction of photons escaping at right angles with increasing impact parameter. As was shown in the previous section (also see Table 1), this is accompanied by higher degrees of polarization. However, when the systems are symmetrical, their net polarization cancels out, as illustrated in the lower part of panel (a) in Figure 2.

This brings us to our next important realization: on a macroscopic, global level, scattering through a geometrically asymmetric system can result in polarization, as was found by Angel (1969) and Lee \& Ahn (1998). This polarization is detectable even without spatially resolving the system. We have shown this by introducing ellipticity, biconical outflows, and central disklike obscuration. In panels (b)-(d) of Figure 2, we display examples of such asymmetric systems and their overall polarization signatures. A spatially averaged polarization signal requires, in the first place, that the local radiation field is polarized. The global polarization orientation is then tangential to the source and reflects the location of the asymmetric regions that provide the surplus of polarized photons. For example, in the bipolar outflow, they emerge from the cones, and the polarization direction is consequently perpendicular to the outflow direction (see Section 3.3). This is similar to the observations of the Egg nebula by You et al. (2017). When the local radiation fields were isotropized by a high number of core scatterings, we found, as did Lee (1994) and Dijkstra \& Loeb (2008), that this reduced the emergent polarization. An example of this is an outflowing, oblate system, in which an increased column density could reduce the polarization, as well as flip the polarization vector (see Figure 5(b)). The same effect occurs in the multiphase systems studied (Section 3.4); i.e., several core scatterings lead to a decrease in polarization, as also identified by Dijkstra \& Loeb (2008) in the context of intergalactic propagation.

The above asymmetries are purely geometrical. It is also possible to introduce asymmetries in velocity space, since a velocity field can lower the (frequency-dependent) optical depth of a system with similar effects as described above. Examples are the ellipsoids presented in Section 3.1 that became more strongly polarized in the presence of global outflows and the outflows in the biconical structures in Section 3.3 that allowed for scattering in them. 
II. Introduced asymmetries. These occur when observing a patch of a larger geometry (intentionally or unintentionally), that is, by effectively masking out regions that would alter the observable. This is illustrated by the sketch in Figure 2(a). With a slit or aperture covering the entire system, one would detect no polarization, as the symmetric polarization vectors cancel out (illustrated in the lower panel). However, if we observe part of the system through a narrow slit, then polarization contributions outside the slit are removed, which breaks the symmetry and yields a surplus of polarization perpendicular to the slit alignment direction. This would result in a global (but possibly misleading) polarization signal. Of course, these issues are less important in imaging polarimetry when one can obtain Stokes parameters on a per-pixel basis (as in Hayes et al. 2011, Prescott et al. 2011, and You et al. 2017).

\subsection{Polarization: Breaking Degeneracies}

We have shown in the results that polarization signals themselves can be degenerate; i.e., several setups can produce similar polarization signatures. A prime example of this is the global polarization signals obtained from nonstatic asymmetric scattering geometries (our ellipsoids; see Figure 5(b)). Here the polarization angle flips from being perpendicular to the major axes of the system to being parallel to them when the column density is increased. Also, when further thinning the medium by introducing outflows, the degree of polarization decreases at higher column densities, compared to an increase with lower column densities.

The origin of this flip is the change of apparent geometry in different column densities (the transition from panel (b) to panel (c) in Figure 2): by lowering the optical depth, one also transitions from escaping and scattering mainly along the minor axis to scattering and escaping in the full system. In the latter case, a surplus of photons escapes from the extended lobes, being polarized tangentially to the source and perpendicular to the major axes.

Without knowledge of the apparent geometry of a system (which is generally the case), we cannot solely use the degree and direction of polarization to constrain the major axis of the system (compare the case $N_{\mathrm{HI}}^{(c)}=10^{19} \mathrm{~cm}^{-2}$ and $v_{\exp }=0 \mathrm{~km} \mathrm{~s}^{-1}$ to that of the rotated system with $N_{\mathrm{HI}}^{(c)}=10^{21} \mathrm{~cm}^{-2}$ and $v_{\exp }=200 \mathrm{~km} \mathrm{~s}^{-1}$ in Figure 5(b)). Similarly, we cannot differentiate between a strong bipolar flow-like geometry, in which the polarization arises due to scattering in the lobes, and a more compact, slightly asymmetric system with obscuration of the core (Section 3.1 compared to Section 3.3). In both cases, we would have a polarization signal aligned with the major axis of the system.

However, similar degeneracies also exist when using other observables. The spectrum is most sensitive to the properties of the scattering medium along the path of least resistance. The ellipsoids explored in Sections 3.1 and 3.2 show examples of this: the spectra do not change when the system changes from being viewed face-on to edge-on. In addition, the Ly $\alpha$ spectra do not necessarily reveal intrinsic dynamics of the scattering gas. Scattering through outflows gives rise to asymmetric spectra, often with a negligible blue peak, as in Figures 5(a) and 8. In the same figures, and in Figure 4(a), we have plotted vertical dashed gray lines, which mark the range of frequencies that could be suppressed by scattering in the IGM (Dijkstra

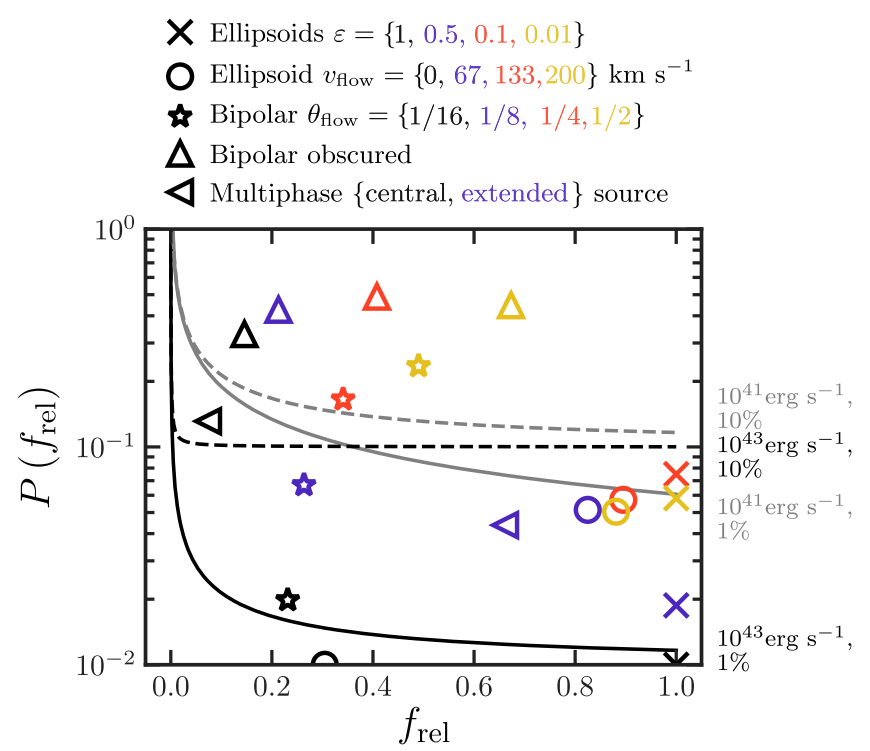

Figure 10. Detectability of models studied in this work. We show the degree of polarization at those frequencies/radii where $P$ differs among them (with $f_{\text {rel }}$ giving the fraction of the total flux for these; see Section 4.3). The solid and dashed lines show the $1 \sigma$ detection limit for a $z \sim 3$ source observed for $1 \mathrm{hr}$ with a VLT-like telescope. We show the impact of a $1 \%(10 \%)$ systemic error and a different luminosity, both shown as labels on the right-hand side of the plot.

et al. 2007; Laursen et al. 2011) and leave a spectral signature virtually identical to that generated by scattering through an optically thick, outflowing medium. This illustrates that degeneracies can exist when using spectra alone.

A joint analysis of Ly $\alpha$ observables can break the mentioned degeneracies: the spectrum can constrain the column density of the optically thinner minor axis. We can then use polarization to constrain the orientation of the system. This allows us, for instance, to differentiate between the polarization signals from static, asymmetric systems and those of dynamic, possibly geometrically symmetrical systems. In static gas geometries, scattering gives rise to symmetric spectra, whereas scattering through dynamic gas geometries generally gives rise to asymmetries in the spectra. Another example relates to which process removes flux blueward of a galaxy's systemic velocity in Ly $\alpha$ spectra: IGM or outflows. The IGM can transform an intrinsically double-peaked profile emerging from a static medium into a spectrum with a dominant red peak and can therefore mimic the effect of scattering through a galactic outflow. However, with only outflows, the polarization increases with offset from the line center (as seen in Figures 5(a) and 8), while it does not in static systems (see Figure 4(a)). A static system with the IGM processing away the blue peak would hence give rise to a different polarization signature. In the case of both IGM processing and outflows, the degree of polarization can attempt to be reconciled with the observed spectral shape.

Finally, in Figure 10, we quantify the ability of present-day telescopes to differentiate between the polarization signatures of all of our explored models. In order to do this, we define the fraction of the total flux $f_{\text {rel }}$ where the polarization signal differs. This fraction can be defined spatially (e.g., only photons arriving in the outer regions for the multiphase media 
in Section 3.4) or in frequency space. This corresponds to an optimally designed experiment where, for instance, the slit position has been chosen so that only photons with a positive net polarization are recorded. Specifically, we then the show the degree of polarization for this fraction of photons $P\left(f_{\text {rel }}\right)$ versus $f_{\text {rel }}$ in Figure 10.

For the outflowing ellipsoids and bipolar outflows (unobscured and obscured), $f_{\text {rel }}$ is obtained for $v>160 \mathrm{~km} \mathrm{~s}^{-1}$ (also indicated by vertical dashed gray lines in the spectra in Figures 5(a) and 8), where the polarization differs the most. For the static ellipsoids, the polarization differs similarly across the spectrum, and all frequencies are included, hence $f_{\text {rel }}=1$ for those. We used photons arriving from $r>0.1 R_{\max }$ for the multiphase media with either a central source of Ly $\alpha$ or sources extending throughout the medium, as the frequency-dependent polarizations for both those models were near zero. With the solid lines, we show the sensitivity ${ }^{19}$ of FORS2 at VLT. We see that it would be able to differentiate between most models if these were $L=10^{43} \mathrm{erg} \mathrm{s}^{-1}$ - or even $L=10^{41} \mathrm{erg} \mathrm{s}^{-1}$ - Ly $\alpha$ emitters at $z=3$ that were observed for $1 \mathrm{hr}$. Separating models becomes harder if one assumes that the systemic error of the instrument is as high as $10 \%$, except for those models where $P$ (frel) $>20 \%$. Detecting, differentiating $P$, and breaking the otherwise degenerate models we have explored is thus already viable today.

\section{Summary and Conclusions}

A major challenge in extragalactic astrophysics is to decode and reveal the properties of systems with only a limited set of observables. Interpreting observations of $\operatorname{Ly} \alpha$ requires us to understand the transport of this radiation. Spectra and/or surface brightness profiles provide constraints on this scattering process, though sometimes not uniquely so. The polarization properties of $\operatorname{Ly} \alpha$ provide additional constraints on the scattering process, but this has been explored much less in the literature, even though it has been demonstrated that extragalactic sources of Ly $\alpha$ can reach significant degrees of polarization (both theoretically and observationally).

This motivated us to implement polarization into the Ly $\alpha$ radiative transfer code tlac of Gronke \& Dijkstra (2014), providing us with the ability to do a joint analysis of emergent observables. To this aim, we used the density matrix formalism of Lee et al. (1994). Through a $2 \times 2$ matrix, it properly describes the probability of measuring a $\operatorname{Ly} \alpha$ photon in either of its two helical spin states, as well as the linear superposition of these. The elements of this are modified through ( $\operatorname{Ly} \alpha)$ core scatterings near the line center and wing scatterings in the damping wing. As photons escape from an arbitrary threedimensional $\mathrm{H}$ I scattering medium that contains a single or a distribution of Ly $\alpha$ sources, we convert the density matrix coefficients of each individual photon to observable Stokes parameters. This approach allows us to treat polarization both on a quantum mechanical level and on a statistical, observable level, setting this work apart from earlier works, where only the latter could be achieved (as in Rybicki \& Loeb 1999, Dijkstra \& Loeb 2008, and Trebitsch et al. 2016).

\footnotetext{
19 We restrict ourselves to a shot-noise/systemic-limited approximation $\sigma_{P}=\sqrt{1 /\left(\left(N_{\mathrm{HWP}} / 2\right) \mathrm{SNR}\right)^{2}+\sigma_{\text {syst }}^{2}}$, where $N_{\mathrm{HWP}}=4$ is the number of halfwave plate rotations, $\mathrm{SNR}=\sqrt{N_{\text {phot }}}$ is the signal-to-noise ratio given in the case of shot noise only from the number of photons $N_{\text {phot }}$ arriving at the sensor, and $\sigma_{\text {syst }}$ is a systemic error (based on Patat \& Romaniello 2006).
}

We have explored scattering through a suite of simplified geometries with simplistic dynamics, such as static and expanding ellipsoids, biconical outflows, and multiphase (clumpy) outflows. We summarize some of their observable properties in Table 2. These idealized models help in understanding the physical origins of the polarization signal and correspond to simplified setups for which other Ly $\alpha$ observables have been studied previously in the literature. We have shown how the global signal from an unresolved source, either its degree and angle of polarization or its polarization spectrum, depends on its scattering symmetry. A symmetric system would appear to have zero polarization, just as one that is either sufficiently optically thick to isotropize the emergent radiation or emits Ly $\alpha$ extensively throughout itself (as in cooling systems, or in the case of recombinations/fluorescence) would have.

By introducing asymmetries in the scattering geometry, from the smallest to the largest scales, we showed how polarization is generated. The polarization is a measure of any surplus or lack of scattered photons at locations for which the scattering geometry is not fully symmetric. We explored ellipsoids and bipolar outflows as examples of this.

The polarization signal-just as other observables — cannot be used alone to describe the physical state of a source and its environment. We have shown that this is only possible when it is used in conjunction with other observables. As an example, we have shown that we obtain tangential polarization patterns around central sources. In asymmetric geometries, the polarization direction may be used to reveal the alignment of the system. But this only works if the intensity spectrum of the system is known, as the polarization direction is degenerate between several geometries and dynamics.

Likewise, other observables should be used with caution. Knowing an intensity spectrum or luminosity of a source, one may misinterpret these as being intrinsic to the source, although in an asymmetric system with anisotropic Ly $\alpha$ escape, it is not necessarily so. The emergent intensity only reveals the properties of the medium along the path of least resistance. With polarization arising due to asymmetries, a global polarized Ly $\alpha$ signal of an unresolved source would be a strong indicator of possible anisotropies in the Ly $\alpha$ escape. In systems with anisotropic Ly $\alpha$ escape, it is easy to misinterpret this as a low-Ly $\alpha$ (and, in some cases, LyC) escape fraction. Moreover, IGM absorption manifests itself through an attenuation of the blue part of the intensity spectra of sources, but this could also be falsely taken to be caused by outflows, which also imprint this spectral signature. We have shown that it is possible to break this degeneracy with polarization measurements.

Currently, none of the next-generation extremely large telescopes plan to include polarimeters intended for extragalactic use (see discussion in Hayes \& Scarlata 2011). However, new, dedicated observations are being undertaken (see, e.g., Beck et al. 2016; You et al. 2017), promising a bright future. We have also shown that present-day telescopes would be able to differentiate between the polarization signals of most of the models we have explored. We will explore a realistic, multiphase medium in an upcoming paper, comparing it to recently obtained observations. Continued work is needed theoretically (see, e.g., Chang et al. 2017), numerically, and observationally, as we have shown in this paper that polarized $\operatorname{Ly} \alpha$ can be a 
Table 2

Intensity and Polarization Properties of Ly $\alpha$ Systems Explored in This Paper

\begin{tabular}{|c|c|c|c|c|c|c|c|c|}
\hline & \multicolumn{2}{|c|}{ Section 3.1 Ellipsoid ${ }^{\mathrm{a}}$} & \multicolumn{2}{|c|}{$\begin{array}{c}\text { Section } 3.2 \text { Ellipsoidal } \\
\text { Outflow }^{\mathrm{a}}\end{array}$} & \multicolumn{2}{|c|}{ Section 3.3 Bipolar Outflow ${ }^{a}$} & \multicolumn{2}{|c|}{ Section 3.4 Multiphase Medium ${ }^{\mathrm{b}}$} \\
\hline & Spherical & Ellipsoidal & Low $v_{\text {flow }}$ & High $v_{\text {flow }}$ & Small $\theta_{\text {flow }}$ & Large $\theta_{\text {flow }}$ & Central Source & Extended Source \\
\hline$I(v)$ & \multicolumn{2}{|c|}{ Symmetric double-peaked } & \multicolumn{2}{|c|}{ Redshifted single-peaked } & \multicolumn{2}{|c|}{ Redshifted doubled-peaked } & \multicolumn{2}{|c|}{ Broad, single peak } \\
\hline$\langle P\rangle^{\mathrm{c}}$ & Zero $^{\mathrm{d}}$ & $\sim 5 \%$ & \multicolumn{2}{|c|}{ Depends on $N_{\mathrm{H} \text { I }}$} & \multicolumn{2}{|c|}{$1 \% \quad 10 \%$} & Zero $^{\mathrm{d}}$ & $<5 \%$ also locally \\
\hline$P(v)$ & Flat, nil & Flat, nonzero & Rises & $\sim 30 \%$ & Flat, low & Rises up to $60 \%$ & \multicolumn{2}{|c|}{ Flat, zero } \\
\hline$P(r)$ & \multicolumn{2}{|c|}{ Rising } & \multicolumn{2}{|c|}{ Rising } & \multicolumn{2}{|r|}{ Rising } & Rising & Flat, zero \\
\hline
\end{tabular}

Notes. The exact numerical values are model-dependent.

${ }^{\text {a }}$ Viewed edge-on.

${ }^{\mathrm{b}}$ Our clumpy clouds have a covering fraction close to the critical value, $f_{\mathrm{c}} \sim f_{\mathrm{c} \text {, crit }}$. Other $f_{\mathrm{c}}$ produce different spectra.

c The globally, frequency-, and spatially integrated polarization values are given as the extremes obtained for the models.

d The polarization is locally nonzero, however. Global symmetries cancel it out, as illustrated in panel (a) of Figure 2.

e These increases in $P$ with $v$ depend on the column density of the system and are here given for $N_{\mathrm{H} \mathrm{I}}=10^{19} \mathrm{~cm}^{-2}$ along the minor axes.

powerful, degeneracy-breaking probe into an otherwise secretive universe.

We all thank B. Ciardi for helpful comments. We thank the referee for the highly constructive feedback. MBE is grateful to H.-W. Lee, S.-J. Chang, C. Scarlata, C. You, and Ll.-M. Ribas, along with other members of the observational and theoretical Ly $\alpha$ community (you know who you are) for rewarding discussions. MBE thanks the Institute of Theoretical Astrophysics at UiO and the Astronomy and Astrophysics Department at UCSC for their kind hospitality. MD thanks the physics department at UCSB for their kind hospitality. MH acknowledges the support of the Swedish Research Council, Vetenskapsrådet, and the Swedish National Space Board (SNSB) and is Fellow of the Knut and Alice Wallenberg Foundation.

Software: tlac (Gronke \& Dijkstra 2014), numpy (Van Der Walt et al. 2011), Cython (Behnel et al. 2011), Matplotlib (Hunter 2007).

\section{Appendix A}

\section{Density Matrices for $\mathrm{Ly} \alpha$ Transitions}

Here we will present the elements of the $2 \times 2$ density matrices describing the probability of measuring the polarization state of a photon following scattering through the three possible Ly $\alpha$ transitions, following Ahn et al. (2002) or Ahn \& Lee (2015).

In the following, we use the left-handed photon basis $\left\{\varepsilon_{1}, \varepsilon_{2}, \varepsilon_{3}\right\}$, where

$$
\begin{gathered}
\varepsilon_{1}=(-\sin \phi, \cos \phi, 0), \\
\varepsilon_{2}=(\cos \theta \cos \phi, \cos \theta \sin \phi,-\sin \theta), \\
\varepsilon_{3}=(\sin \theta \cos \phi, \sin \theta \sin \phi, \cos \theta),
\end{gathered}
$$

with $\phi$ and $\theta$ as the polar angles.

The state of a Ly $\alpha$ photon after scattering is determined by eight unique photon parameters: (i) the photon frequency, determining whether the transition occurs in the resonance core or the damping wing, and hence the transition type; (ii)-(iii) the two polar angles $\theta$ and $\phi$, uniquely specifying the photon orientation prior to scattering; (iv)-(v) the polar angles $\theta^{\prime}$ and $\phi^{\prime}$ following a scattering; and (vi)-(viii) the density matrix of the unscattered photon $\rho_{\text {phot }}$ with unique elements $\rho_{\text {phot } 11}, \rho_{\text {phot }, 12}=\rho_{\text {phot }, 21}^{*}=\rho_{\text {phot }, 21}, \rho_{\text {phot }, 22}$. A difference in angles is given as $\Delta \phi=\phi^{\prime}-\phi$. The state of the photon after a scattering can then be represented by a new density matrix $\rho_{\text {phot }}^{\prime}$.

For H-type resonance core scatterings through the $2 P_{1 / 2}$ state of $\mathrm{HI}$, in which the wave function of the hydrogen atom has no angular dependence, the photon is depolarized following a scattering event, and the resulting density matrix elements are

$$
\rho_{11}^{\prime}=\rho_{22}^{\prime}=1 / 2 ; \quad \rho_{12}^{\prime}=0 .
$$

For K-type resonance core scatterings through the $2 P_{3 / 2}$ state, there is a strong angular dependence and polarization state of the incoming photon,

$$
\begin{aligned}
\rho_{11}^{\prime}= & (5+3 \cos 2 \Delta \phi) \rho_{11}-6 \cos \theta \sin 2 \Delta \phi \rho_{12} \\
& +\left[(5-3 \cos 2 \Delta \phi) \cos ^{2} \theta+2 \sin ^{2} \theta\right] \rho_{22},
\end{aligned}
$$

$$
\begin{aligned}
\rho_{12}^{\prime}= & 3 \sin 2 \Delta \phi \cos \theta^{\prime} \rho_{11} \\
& +6\left[\cos \theta \cos \theta^{\prime} \cos 2 \Delta \phi+\sin \theta \sin \theta^{\prime} \cos \Delta \phi\right] \rho_{12} \\
& +3 \cos \theta\left(-2 \sin \theta \sin \theta^{\prime} \sin \Delta \phi\right. \\
& \left.-\cos \theta \cos \theta^{\prime} \sin 2 \Delta \phi\right) \rho_{22},
\end{aligned}
$$

$$
\begin{aligned}
\rho_{22}^{\prime}= & {\left[(5-3 \cos 2 \Delta \phi) \cos ^{2} \theta^{\prime}+2 \sin ^{2} \theta^{\prime}\right] \rho_{11} } \\
& +\left[(5+3 \cos 2 \Delta \phi) \cos ^{2} \theta \cos ^{2} \theta^{\prime}\right. \\
& +2 \cos ^{2} \theta \sin ^{2} \theta^{\prime} \\
& +12 \cos \Delta \phi \cos \theta^{\prime} \cos \theta \sin \theta \sin \theta^{\prime} \\
& \left.+2 \cos ^{2} \theta^{\prime} \sin ^{2} \theta+8 \sin ^{2} \theta \sin ^{2} \theta^{\prime}\right] \rho_{22} \\
& +\left(6 \sin 2 \Delta \phi \cos \theta \cos ^{2} \theta^{\prime}\right. \\
& \left.+2 \sin \Delta \phi \cos \theta^{\prime} \sin \theta \sin \theta^{\prime}\right) \rho_{12} .
\end{aligned}
$$

For wing scatterings, in which the Stenflo (1980) effect effectively makes the transition behave as scattering off a free electron, the resulting density matrix elements are

$$
\rho_{11}^{\prime}=\cos ^{2} \phi \rho_{11}-\cos \theta \sin 2 \Delta \phi \rho_{12}+\cos ^{2} \theta \sin ^{2} \Delta \phi \rho_{22}
$$




$$
\begin{aligned}
\rho_{12}^{\prime}= & 1 / 2 \cos \theta^{\prime} 2 \Delta \phi \rho_{11} \\
& +\left[\cos \theta \cos \theta^{\prime} \cos 2 \Delta \phi+\sin \theta \sin \theta^{\prime} \cos \Delta \phi\right] \rho_{12} \\
& -\cos \theta\left(\sin \theta \sin \theta^{\prime} \sin \Delta \phi\right. \\
& \left.+1 / 2 \cos \theta \cos \theta^{\prime} \sin 2 \Delta \phi\right) \rho_{22}, \\
\rho_{22}^{\prime}= & \cos ^{2} \theta^{\prime} \sin ^{2} \Delta \phi \rho_{11} \\
& +\cos \theta^{\prime}\left[2 \sin \theta \sin \theta^{\prime} \sin \Delta \phi+\cos \theta \cos \theta^{\prime} \sin 2 \Delta \phi\right] \rho_{12} \\
& +\left(\cos \theta \cos \theta^{\prime} \cos \Delta \phi+\sin \theta \sin \theta^{\prime}\right)^{2} \rho_{22} .
\end{aligned}
$$

\section{Appendix B \\ Translating to an Observer-specific Coordinate System}

The polarization vectors $\varepsilon_{1}$ and $\varepsilon_{2}$ chosen by Ahn \& Lee (2015) were specific for a semi-infinite planar slab in the $x, y$ directions. The density matrix elements are composed of the (possibly complex) coefficients $c_{1}$ and $c_{2}$, which were estimated for the chosen polarization vectors $\varepsilon_{1}$ and $\varepsilon_{2}$, which are also given in Equations (15) and (16).

For a more general case, it can be useful to translate these coordinate-specific coefficients to a more general geometry. Writing the polarization state vector as $\boldsymbol{P}$ in terms of its components $\varepsilon_{1}$ and $\varepsilon_{2}$,

$$
\begin{aligned}
\boldsymbol{P}= & c_{1} \varepsilon_{1}+c_{2} \varepsilon_{2}=c_{1}\left(-\sin \phi \boldsymbol{e}_{x}+\cos \phi \boldsymbol{e}_{y}\right) \\
& +c_{2}\left(\cos \theta \cos \phi \boldsymbol{e}_{x}+\cos \theta \sin \phi \boldsymbol{e}_{y}-\sin \theta \boldsymbol{e}_{z}\right) \\
= & \left(-c_{1} \sin \phi+c_{2} \cos \theta \cos \phi\right) \boldsymbol{e}_{x} \\
& +\left(c_{1} \cos \phi+c_{2} \cos \phi \sin \phi\right) \boldsymbol{e}_{y}-c_{2} \sin \theta \boldsymbol{e}_{z},
\end{aligned}
$$

allows us to instead express the polarization state vector $\boldsymbol{P}$ in terms of the Cartesian components with related coefficients:

$$
\begin{gathered}
c_{x}=-c_{1} \sin \phi+c_{2} \cos \theta \cos \phi, \\
c_{y}=c_{1} \cos \phi+c_{2} \cos \theta \sin \phi, \\
c_{z}=-c_{2} \sin \theta .
\end{gathered}
$$

We can now construct an observer-specific density matrix relative to the three Cartesian unit vectors,

$$
\rho_{\mathrm{obs}}=\boldsymbol{P P}^{\dagger}=\left(\begin{array}{cccc}
c_{x} c_{x}^{*} & c_{x} c_{y}^{*} & c_{x} c_{z}^{*} \\
c_{y} c_{x}^{*} & c_{y} c_{y}^{*} & c_{y} c_{z}^{*} \\
c_{z} c_{x}^{*} & c_{z} c_{y}^{*} & c_{z} c_{z}^{*}
\end{array}\right),
$$

which can be expressed in terms of the coefficients $c_{1}$ and $c_{2}$,

$$
\begin{aligned}
\left|c_{x}^{2}\right|=\mid & \left|c_{1}^{2}\right| \sin ^{2} \phi-\left(c_{1} c_{2}^{*}+c_{2} c_{1}^{*}\right) \sin \phi \cos \theta \cos \phi \\
& +\left|c_{2}^{2}\right| \cos ^{2} \theta \cos ^{2} \phi, \\
\left|c_{y}^{2}\right|=\mid & \left|c_{1}^{2}\right| \cos ^{2} \phi+\left(c_{1} c_{2}^{*}+c_{2} c_{1}^{*}\right) \cos \phi \cos \theta \sin \phi \\
& +\left|c_{2}^{2}\right| \cos ^{2} \theta \sin ^{2} \phi, \\
& \left|c_{z}^{2}\right|=\left|c_{2}^{2}\right| \sin ^{2} \theta, \\
c_{x} c_{y}^{*}= & -\left|c_{1}^{2}\right| \sin \phi \cos \phi-c_{1} c_{2}^{*} \sin \phi \cos \theta \sin \phi \\
& +c_{2} c_{1}^{*} \cos \theta \cos ^{2} \phi \\
& +\left|c_{2}^{2}\right| \cos ^{2} \theta \cos \phi \sin \phi,
\end{aligned}
$$

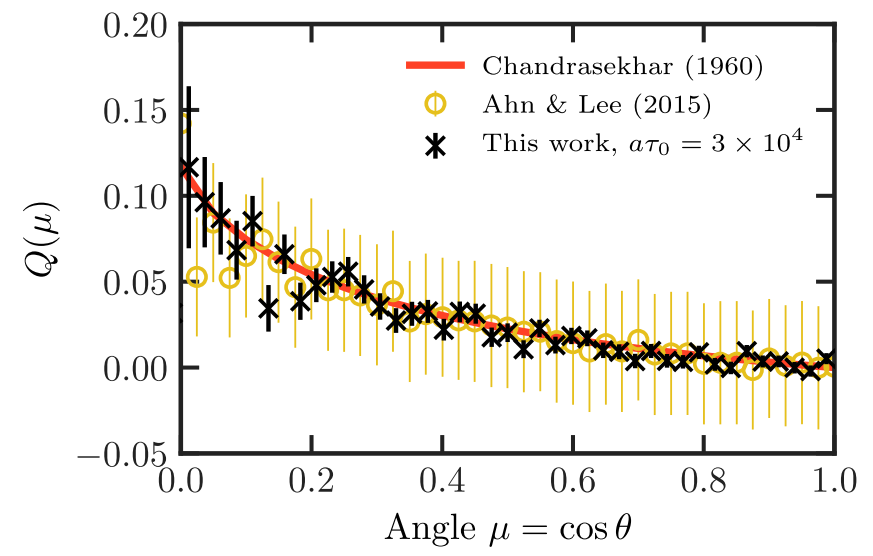

Figure 11. Degree of $Q$-polarization calculated in a semi-infinite plane-parallel slab of neutral hydrogen with $T=10 \mathrm{~K}$ and $\tau_{0}=2 \times 10^{6}$ and the similar calculations by Ahn \& Lee (2015), who introduced the implemented density matrix formalism, and an analytical solution by Chandrasekhar (1960) for electron scatterings in an optically thick slab, showing good agreement.

$$
\begin{gathered}
c_{x} c_{z}^{*}=c_{1} c_{2}^{*} \sin \phi \sin \theta-c_{2} \cos \theta \cos \phi \sin \theta, \\
c_{y} c_{z}^{*}=-c_{1} c_{2}^{*} \cos \phi \sin \theta-\left|c_{2}^{2}\right| \cos \theta \sin \phi \sin \theta, \\
c_{x}^{*} c_{y}=\left(c_{x} c_{y}^{*}\right)^{*}, c_{x}^{*} c_{z}=\left(c_{x} c_{z}^{*}\right)^{*}, c_{y}^{*} c_{z}=\left(c_{y} c_{z}^{*}\right)^{*},
\end{gathered}
$$

that can be retrieved directly from the original density matrix's components,

$$
\left|c_{1}^{2}\right|=\rho_{11}, c_{1} c_{2}^{*}=\rho_{12}, c_{1}^{*} c_{2}=\rho_{21},\left|c_{2}^{2}\right|=\rho_{22},
$$

where $\rho_{12}=\rho_{21}$ when there is no circular polarization $(V=0$, and hence all elements are real).

\section{Appendix C Comparisons}

\section{C.1. Semi-infinite Slab}

To test our implementation of the density matrix formalism in tlac, we compare it under the same geometry that was applied by Ahn \& Lee (2015). In Figure 11, we show results for a slab that is infinitely large in the $x, y$ directions. This orientation ensures that it is aligned with the basis chosen to represent the density matrix. The observable Stokes parameters do, in this case, coincide with the per-photon Stokes parameters. These can be obtained from the density matrix components, with $I=\rho_{11}+\rho_{22}, \quad Q=\rho_{11}-\rho_{22}, \quad$ and $U=$ $\rho_{12}+\rho_{21}$. Hence, we do not have to translate the components as in Appendix B. For the semi-infinite slab, polarization may only develop either parallel to the slab normal or perpendicular to it, which corresponds to the two basis vectors. No crossterms then exist, and $U$ is zero. In the figure, we then only plot $Q$, as a function of viewing angle $\mu=\cos \theta$ to the surface, where $\mu=1$ corresponds to viewing the slab nadir. The optical depth from the midplane of the slab is $2 \times 10^{6}$, and $T=10 \mathrm{~K}$. This corresponds to the optically thick limiting case in which the photons escape from the damping wing after having undergone a diffusion process. There, the polarization is given by the Rayleigh phase function, in which the polarization develops similarly to a process where a continuum photon is scattered by a free electron, for which Chandrasekhar (1960) developed a solution, overplotted in the figure. Our results fit those of Chandrasekhar (1960) and Ahn \& Lee (2015) well, 

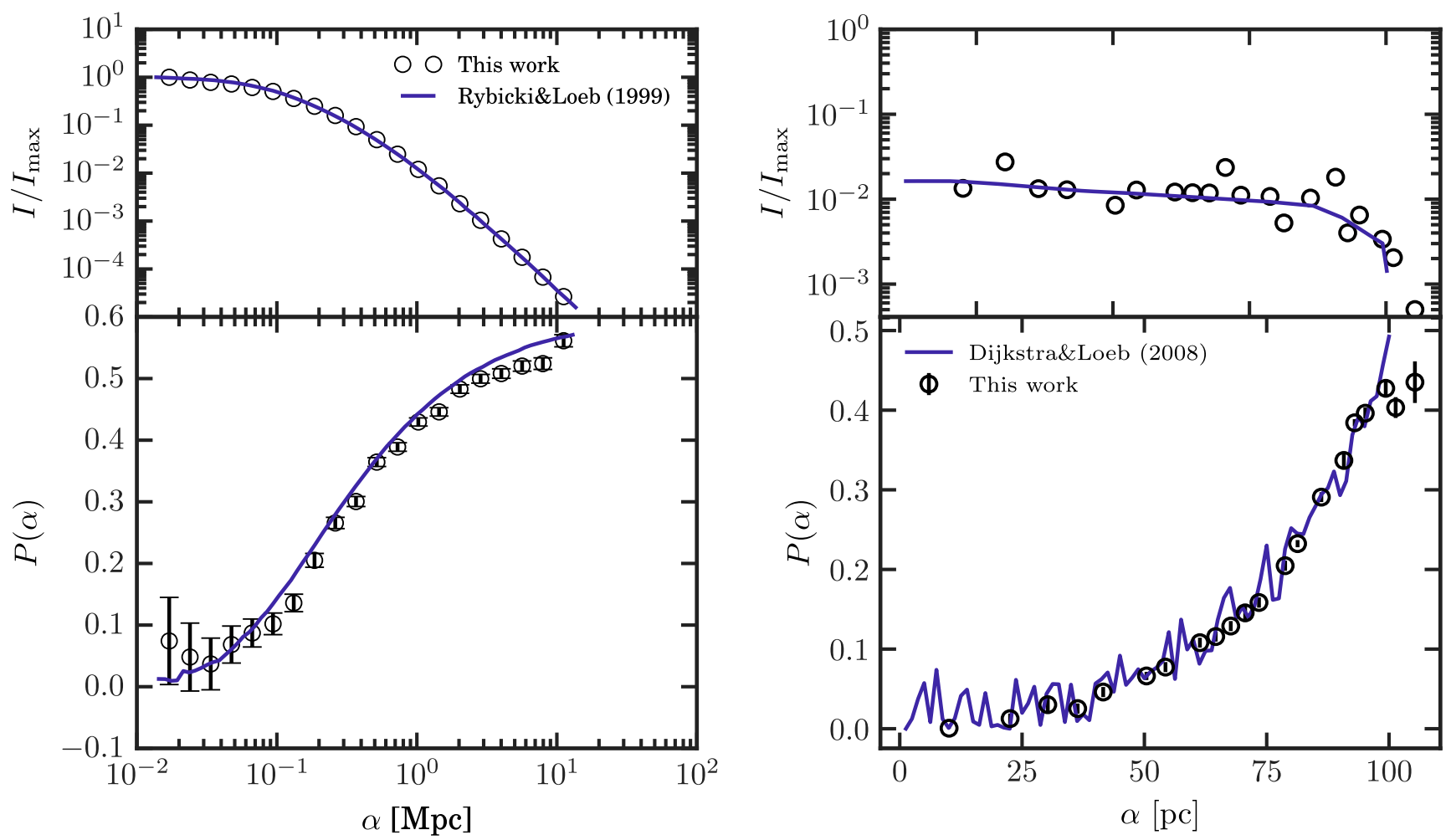

Figure 12. Upper panels: normalized intensity as a function of impact parameter. Lower panels: degree of linear polarization against impact parameter. In the left panels, we compare our methods against the results of Rybicki \& Loeb (1999; solid purple lines) for scattering in a Hubble-expanding medium of H I at $z=10$, and in the right panels, we compare our methods (open black circles with error bars for $P(\alpha)$ ) against the results for an expanding shell of $\mathrm{H}$ I by Dijkstra \& Loeb (2008; solid purple lines).

with a larger polarization developing for sharper viewing angles $(\mu \rightarrow 0)$.

\section{C.2. Universe before Reionization: Scattering Out of Resonance Due to the Hubble Expansion}

We compare our polarization implementation with that of Rybicki \& Loeb (1999), who (also through the companion paper Loeb \& Rybicki 1999) examined the scattering of Ly $\alpha$ photons through neutral hydrogen in an expanding Hubble volume, representative of the high- $z$, pre-reionization universe. They predicted that $\operatorname{Ly} \alpha$ is detectable as halos with a characteristic radius of $\sim 10-20^{\prime \prime}$ and redshifted by several $10^{3} \mathrm{~km} \mathrm{~s}^{-1}$ with respect to systemic due to the Hubble flow (the precise numbers depend on the cosmological parameters). Rybicki \& Loeb (1999) computed the polarization properties of these Ly $\alpha$ halos and found them to be highly polarized, with the degree of linear polarization reaching $\sim 60 \%$. Rybicki \& Loeb (1999) compared their results to those of Schuster (1879), where the polarization was directly related to the radial dependence of the product of the cross section and the column density. We, however, note that the degree of polarization is consistent with photons that have developed $100 \%$ polarization in an early wing scattering that then is core-scattered forward (see Table 1).

To test our polarization implementation, we repeated the analysis of Rybicki \& Loeb (1999) and compared the results. We adopted $\Omega_{\mathrm{b}}=0.06, \Omega_{\mathrm{m}}=0.7, \Omega_{\Lambda}=0.7$, and $h_{0}=0.7$, which translates to a mean present-day hydrogen number density $n_{\mathrm{H}, 0}=x_{\mathrm{H}} X n_{\mathrm{b}}$, where $n_{\mathrm{b}}=2.5 \times 10^{-7} \mathrm{~cm}^{-3}$ (Planck Collaboration XVI 2014), $x_{\mathrm{H}_{\mathrm{I}}}$ is the neutral hydrogen fraction, and $X$ is the primordial number density of hydrogen, giving
$n_{\mathrm{H}}(z)=n_{\mathrm{H} \mathrm{I}, 0}(1+z)^{3}$. Following Loeb \& Rybicki (1999) and Rybicki \& Loeb (1999), we assumed that $x_{\mathrm{H}}=1$ and focused on $z=10$. We emitted $10^{7}$ photons centered at the Ly $\alpha$ line frequency and set the core-skipping parameter $x_{\text {crit }}=10$. We captured all photons escaping within $15^{\circ}$ of the six principal axes and binned the photons into logarithmic bins of their impact parameters. We then calculated the collective degree of linear polarization in each bin, $P\left(\alpha_{x}, \alpha_{y}\right)$, where $\alpha_{x}$ and $\alpha_{y}$ are their two-dimensional impact parameters, as well as the variance. We calculated the uncertainty following the procedure outlined in Appendix D. We then computed the photonweighted averages of the linear polarization, $P(\alpha)$, where $\alpha$ is now the radial impact parameter.

Figure 12(a) compares our results to those of Rybicki \& Loeb (1999). We plot our normalized intensity $I / I_{\max }$ and the degree of total linear polarization $P(\alpha)$ as a function of the radial impact parameter $\alpha$ (open black circles with error bars), compare to the results from Figure 1 in Rybicki \& Loeb (1999), and rescale their normalized impact parameter $\tilde{p}$ using Equation (9) in Loeb \& Rybicki (1999) with our choice of cosmological parameters. We find that our results fit the rescaled results of Rybicki \& Loeb (1999) well. We slightly underestimate $P(\alpha)$ at larger $\alpha$. We attribute the discrepancies in the degree of polarization to the choice of cosmology.

\section{C.3. Scattering in an Ionized Medium Off an Expanding, Neutral Shell of Hydrogen}

Dijkstra \& Loeb (2008) explored the polarization of Ly $\alpha$ photons scattering through the often-used shell models. We repeat their analysis for a shell with column density $N_{\mathrm{H}}=10^{19} \mathrm{~cm}^{-2}$ at $T=10^{4} \mathrm{~K}, v_{\text {outflow }}=200 \mathrm{~km} \mathrm{~s}^{-1}$. In this 
simulation, we include all core scatterings (i.e., $x_{\text {crit }}=0$ ). The central Ly $\alpha$ source emits unpolarized photons with frequencies Gaussian-distributed around the $\operatorname{Ly} \alpha$ line center, with $\sigma=v_{\text {circ }}$. Figure 12(b) compares the rescaled and then normalized intensity $I / I_{\max }$ and the degree of linear polarization $P(\alpha)$. Our results are represented by the open black circles, whereas those of Dijkstra \& Loeb (2008) are represented by the solid purple line. Both results agree well.

\section{Appendix D Estimating Uncertainty}

The polarization in each bin (pixel) is estimated from the contributions from all the photons falling into it. The arrival of photons on a detector is a Poissonian process, which for a large number of photons is well described by Gaussian statistics. The variance can be estimated from the accumulated (or mean) polarization $P_{j}$ in a pixel $j$ and the per-photon polarization $P_{i, j}$,

$$
\sigma_{j}^{2}=\frac{1}{N_{j}-1} \sum_{i=1}^{N_{j}}\left(P_{i, j}-P_{j}\right)^{2} .
$$

To calculate the polarization as a function of the radial impact parameter $\alpha$, we bin the polarization in multiple pixels with (approximately) the same $\alpha$,

$$
P(\alpha)=\frac{\sum_{j \in \alpha} w_{j} P_{j}}{\sum_{j \in \alpha} w_{j}},
$$

where we have weighted the contributions by the number of photons going into each pixel $j, N_{j}$, and the variance $\sigma_{j}^{2}$,

$$
w_{j}=\frac{N_{j}}{\sigma_{j}^{2}}
$$

We calculate the standard error of the mean polarization $P(\alpha)$ by propagating the per-pixel standard error,

$$
\mathrm{SE}(P(\alpha))=\sqrt{\sum_{j \in \alpha} \frac{1}{N_{j}}\left(\frac{\partial P(\alpha)}{\partial w_{j}} \sigma_{j}\right)^{2}}=\frac{1}{\sum_{j \in \alpha} w_{j}} \sqrt{\sum_{j \in \alpha} \frac{N_{j}}{\sigma_{j}^{2}}} .
$$

\section{ORCID iDs}

Marius B. Eide (1) https://orcid.org/0000-0002-4234-2012 Max Gronke (1) https://orcid.org/0000-0003-2491-060X Matthew Hayes (ํ) https://orcid.org/0000-0001-8587-218X

\section{References}

Adams, T. F. 1971, ApJ, 168, 575

Adams, T. F. 1972, ApJ, 174, 439

Ahn, S.-H., \& Lee, H.-W. 1998, arXiv:astro-ph/9801031

Ahn, S.-H., \& Lee, H.-W. 2015, JKAS, 48, 195

Ahn, S.-H., Lee, H.-W., \& Lee, H. M. 2000, JKAS, 33, 29

Ahn, S.-H., Lee, H.-W., \& Lee, H. M. 2003, MNRAS, 340, 863

Ahn, S., Lee, H., \& Lee, H. M. 2002, ApJ, 567, 922

Angel, J. R. P. 1969, ApJ, 158, 219

Avery, L. W., \& House, L. L. 1968, ApJ, 152, 493

Bacon, R., Brinchmann, J., Richard, J., et al. 2015, A\&A, 575, A75

Beck, M., Scarlata, C., Hayes, M., Dijkstra, M., \& Jones, T. J. 2016, ApJ, 818, 1

Behnel, S., Bradshaw, R., Citro, C., et al. 2011, CSE, 13, 31

Blandford, R. D., \& Rees, M. J. 1974, MNRAS, 169, 395

Bower, R. 2011, Natur, 476, 288

Brasken, M., \& Kyrola, E. 1998, A\&A, 332, 732
Cai, Z., Fan, X., Yang, Y., et al. 2017, ApJ, 837, 71

Cantalupo, S., Arrigoni-Battaia, F., Prochaska, J. X., Hennawi, J. F., \& Madau, P. 2014, Natur, 506, 63

Cantalupo, S., Porciani, C., Lilly, S. J., \& Miniati, F. 2005, ApJ, 628, 61

Chandrasekhar, S. 1960, Radiative Transfer (New York: Dover), 393

Chang, S.-J., Lee, H.-W., \& Yang, Y. 2017, MNRAS, 464, 5018

Chapman, S. C., Smail, I., Windhorst, R., Muxlow, T., \& Ivison, R. J. 2004, ApJ, 611, 732

Dijkstra, M. 2014, PASA, 31, 26

Dijkstra, M., Gronke, M., \& Sobral, D. 2016, ApJ, 823, 74

Dijkstra, M., Haiman, Z., \& Spaans, M. 2006, ApJ, 649, 14

Dijkstra, M., \& Kramer, R. 2012, MNRAS, 424, 1672

Dijkstra, M., Lidz, A., \& Wyithe, J. S. B. 2007, MNRAS, 377, 1175

Dijkstra, M., \& Loeb, A. 2008, MNRAS, 386, 492

Djorgovski, S., Spinrad, H., McCarthy, P., \& Strauss, M. A. 1985, ApJL, 299, L1

Duval, F., Östlin, G., Hayes, M., et al. 2016, A\&A, 587, A77

Field, G. B. 1958, PIRE, 46, 240

Gallagher, J. S. I. I. I., \& Smith, L. J. 1999, MNRAS, 304, 540

Geach, J. E., Alexander, D. M., Lehmer, B. D., et al. 2009, ApJ, 700, 1

Gronke, M., Bull, P., \& Dijkstra, M. 2015, ApJ, 812, 123

Gronke, M., \& Dijkstra, M. 2014, MNRAS, 444, 1095

Gronke, M., \& Dijkstra, M. 2016, ApJ, 826, 14

Gronke, M., Dijkstra, M., McCourt, M., \& Oh, S. P. 2016, ApJL, 833, L26

Gronke, M., Dijkstra, M., McCourt, M., \& Oh, S. P. 2017, arXiv:1704.06278

Haiman, Z., Spaans, M., \& Quataert, E. 2000, ApJL, 537, L5

Hansen, M., \& Peng Oh, S. 2006, MNRAS, 367, 979

Hashimoto, T., Ouchi, M., Shimasaku, K., et al. 2017, MNRAS, 465, 1543

Hashimoto, T., Verhamme, A., Ouchi, M., et al. 2015, ApJ, 812, 157

Hayes, M. 2015, PASA, 32, e027

Hayes, M., Östlin, G., Schaerer, D., et al. 2013, ApJL, 765, L27

Hayes, M., \& Scarlata, C. 2011, in SF2A, ed. G. Alecian et al. (Paris: Sociètè Francaise dAstronomie et dAstrophysique), 129

Hayes, M., Scarlata, C., \& Siana, B. 2011, Natur, 476, 304

Hennawi, J. F., Prochaska, J. X., Cantalupo, S., \& Arrigoni-Battaia, F. 2015, Sci, 348, 779

Herenz, E. C., Urrutia, T., Wisotzki, L., et al. 2017, A\&A, 606, A12

Herenz, E. C., Wisotzki, L., Roth, M., \& Anders, F. 2015, A\&A, 576, A115

Hogan, C. J., \& Weymann, R. J. 1987, MNRAS, 225, 1

Hu, E. M., \& Cowie, L. L. 1987, ApJL, 317, L7

Humphrey, A., Vernet, J., Villar-Martín, M., et al. 2013, ApJL, 768, L3

Hunter, J. D. 2007, CSE, 9, 99

Karman, W., Caputi, K. I., Caminha, G. B., et al. 2016, A\&A, 599, A28

Kim, H. J., Lee, H.-W., \& Kang, S. 2007, MNRAS, 374, 187

Kunth, D., Mas-Hesse, J. M., Terlevich, E., et al. 1998, A\&A, 334, 11

Lake, E., Zheng, Z., Cen, R., et al. 2015, ApJ, 806, 46

Laursen, P. 2010, PhD thesis, Univ. Copenhagen

Laursen, P., Duval, F., \& Östlin, G. 2013, ApJ, 766, 124

Laursen, P., Sommer-Larsen, J., \& Razoumov, A. O. 2011, ApJ, 728, 52

Lee, H.-W. 1994, MNRAS, 268, 49

Lee, H.-W. 1999, ApJL, 511, L13

Lee, H.-W., \& Ahn, S.-H. 1998, ApJL, 504, L61

Lee, H.-W., \& Blandford, R. D. 1997, MNRAS, 288, 19

Lee, H.-W., Blandford, R. D., \& Western, L. 1994, MNRAS, 267, 303

Lee, K. W., \& Lee, H.-W. 1997, MNRAS, 292, 573

Loeb, A., \& Rybicki, G. B. 1999, ApJ, 524, 527

Lynds, C. R., \& Sandage, A. R. 1963, ApJ, 137, 1005

Madau, P., Meiksin, A., \& Rees, M. J. 1997, ApJ, 475, 429

Mas-Ribas, L., \& Dijkstra, M. 2016, ApJ, 822, 84

Mas-Ribas, L., Dijkstra, M., Hennawi, J. F., et al. 2017, ApJ, 841, 19

McCourt, M., Oh, S. P., O’Leary, R. M., \& Madigan, A.-M. 2016, arXiv:1610. 01164

McKee, C. F., \& Ostriker, J. P. 1977, ApJ, 218, 148

Mori, M., Umemura, M., \& Ferrara, A. 2004, ApJL, 613, L97

Neufeld, D. A. 1990, ApJ, 350, 216

Neufeld, D. A. 1991, ApJL, 370, L85

Oesch, P. A., Brammer, G., van Dokkum, P. G., et al. 2016, ApJ, 819, 129

Osterbrock, D. E. 1962, ApJ, 135, 195

Ouchi, M., Harikane, Y., Shibuya, T., et al. 2017, arXiv:1704.07455

Partridge, R. B., \& Peebles, P. J. E. 1967, ApJ, 147, 868

Patat, F., \& Romaniello, M. 2006, PASP, 118, 146

Pierleoni, M., Maselli, A., \& Ciardi, B. 2009, MNRAS, 393, 872

Planck Collaboration XVI, 2014, A\&A, 571, A16

Prescott, M. K. M., Smith, P. S., Schmidt, G. D., \& Dey, A. 2011, ApJL, 730, L25

Rybicki, G. B., \& Lightman, A. P. 1979, Radiative processes in Astrophysics (Weinheim: Wiley-VCH), 382 
Rybicki, G. B., \& Loeb, A. 1999, ApJL, 520, L79

Schaerer, D. 2002, A\&A, 382, 28

Schaerer, D. 2003, A\&A, 397, 527

Schuster, A. 1879, MNRAS, 40, 35

Shibuya, T., Ouchi, M., Konno, A., et al. 2018, PASJ, 70, 14

Spinrad, H. 1989, in The Epoch of Galaxy Formation, Vol. 264, ed. C. S. Frenk et al. (Dordrecht: Kluwer), 39

Steidel, C. C., Bogosavljević, M., Shapley, A. E., et al. 2011, ApJ, 736, 160

Stenflo, J. O. 1980, A\&A, 84, 68

Suchkov, A. A., Balsara, D. S., Heckman, T. M., \& Leitherner, C. 1994, ApJ, 430, 511

Taniguchi, Y., \& Shioya, Y. 2000, ApJL, 532, L13

Trainor, R. F., Steidel, C. C., Strom, A. L., \& Rudie, G. C. 2015, ApJ, 809, 89
Trebitsch, M., Verhamme, A., Blaizot, J., \& Rosdahl, J. 2016, A\&A, 593, A122

Van Der Walt, S., Colbert, S. C., \& Varoquaux, G. 2011, CSE, 13, 22

Verhamme, A., Orlitová, I., Schaerer, D., et al. 2017, A\&A, 597, A13

Verhamme, A., Schaerer, D., Atek, H., \& Tapken, C. 2008, A\&A, 491, 89

Verhamme, A., Schaerer, D., \& Maselli, A. 2006, A\&A, 460, 397

Wisotzki, L., Bacon, R., Blaizot, J., et al. 2016, A\&A, 587, A98

Wouthuysen, S. A. 1952, AJ, 57, 31

Yang, H., Malhotra, S., Gronke, M., et al. 2017, ApJ, 844, 171

You, C., Zabludoff, A., Smith, P., et al. 2017, ApJ, 834, 182

Zhang, H., \& Yan, H. 2018, MNRAS, 475, 2415

Zheng, Z., \& Miralda-Escudé, J. 2002, ApJ, 578, 33

Zitrin, A., Labbe, I., Belli, S., et al. 2015, ApJL, 810, L12 